\title{
Computational Aerothermodynamic Design Issues for Hypersonic Vehicles
}

\author{
Peter A. Gnoffo, K. James Weilmuenster, H. Harris Hamilton, II* \\ NASA Langley Research Center \\ Hampton, VA 23681-0001
}

\author{
David R. Olynick ${ }^{\dagger}$ \\ NASA Ames Research Center \\ Ethiraj Venkatapathy \\ Thermosciences Institute \\ Moffett Field, CA 94035-1000 \\ NASA Ames Research Center \\ Moffett Field, CA 94035-1000
}

\begin{abstract}
A brief review of the evolutionary progress in computational aerothermodynamics is presented. The current status of computational aerothermodynamics is then discussed, with emphasis on its capabilities and limitations for contributions to the design process of hypersonic vehicles. Some topics to be highlighted include: (1) aerodynamic coefficient predictions with emphasis on high temperature gas effects; (2) surface heating and temperature predictions for thermal protection system (TPS) design in a high temperature, thermochemical nonequilibrium environment; (3) methods for extracting and extending computational fluid dynamic (CFD) solutions for efficient utilization by all members of a multidisciplinary design team; (4) physical models; (5) validation process and error estimation; and (6) gridding and solution generation strategies. Recent experiences in the design of X-33 will be featured. Computational aerothermodynamic contributions to Mars Pathfinder, METEOR, and Stardust (Comet Sample return) will also provide context for this discussion. Some of the barriers that currently limit computational aerothermodynamics to a predominantly reactive mode in the design process will also be discussed, with the goal of providing focus for future research.
\end{abstract}

\section{Introduction}

A EROTHERMODYNAMICS couples the disciplines of aerodynamics and thermodynamics. It most often deals with problems in hypersonic flight in which high temperature gas effects strongly influence the fluid forces (pressure, skin friction), energy flux (convective and radiative heating), and mass flux (ablation) on a vehicle. Hypersonic flows are usually characterized by the presence of strong shocks and equilibrium or non-equilibrium gas chemistry. Accurate prediction of these effects is critical to the design of any vehicle which flies at hypersonic velocities. Fluid forces are integrated over the complete configuration to define the aerodynamic forces (lift, drag, pitching moment, control surface effectiveness). Peak temperatures, peak heat transfer and heating load (heating rate integrated over time) are mapped over the vehicle surface to design the thermal protection system.

\footnotetext{
* Senior Research Engineer, Associate Fellow AIAA.

$\dagger$ Research Scientist, Senior Member AIAA

$\ddagger$ Senior Research Scientist, Senior Member AIAA

Copyright (C) 1997 by the American Institute of Aeronautics and Astronautics, Inc. No copyright is asserted in the United States under Title 17, U.S. Code. The U.S. Government has a royalty-free license to exercise all rights under the copyright claimed herein for governmental purposes. All other rights are reserved by the copyright owner.
}

Pressure distributions are required for assessment of structural loads and venting environments.

Computational aerothermodynamics uses numerical solutions of the governing equations for continuity, momentum, and energy conservation to model the flow, including appropriate physical models for the high temperature environment. It faces most of the same challenges as computational fluid dynamics regarding cost and simplicity of generating answers to be used in the design process. Surface and volume grids are often difficult and/or time consuming to generate. Perturbations to the configuration are not easily accomodated unless special considerations and foresight were applied in the initial grid generation process. Order $10^{5}$ or greater cells are required to discretize the flowfield around real configurations, and solution of the governing equations requires hours to tens of hours per steady state solution on a CRAY C-90.

Computational aerothermodynamics faces some challenges unique to the high temperature, hypersonic flow environment. Equations for chemical and thermal nonequilibrium, including source terms which may add stiffness to the equation set, must be included. A single case may include flow domains that vary from broad, subsonic, high pressure and temperature stagnation regions behind strong bow shock waves to high 
speed, low density (high Knudsen number) regions in the lee side.

The grand challenge is to implement these solutions on the order of minutes so that they may be an intimate and integral part of the design process. We seem to be a long way from this goal. Structured grid generation for the $\mathrm{X}-33^{1}$ (a configuration with wing, tail, deflected control surfaces extending out over the wake, gaps, etc.) requires two to three weeks of effort, with close collaboration between CFD and grid generation specialists. Unstructured grid generation on this same configuration requires approximately one to three days, but at present is only applicable with confidence to inviscid flow resolution. Thin-layer Navier-Stokes solutions for steady, laminar flow using a five species chemical model requires $\mathrm{O}(10)$ hours to get decent, first cut aerodynamics on a coarsened grid. Converged heating levels on a fine grid generally require a factor of three more relaxation time. We must do better. Some reflections are offered on the current design environment, CFD state-of-art, and the types of advances needed to achieve the target design goals.

\section{Historical Perspective and Technology Drivers}

Driving applications for external, continuum, hypersonic flow simulation in the period 1980 to 1990 in the United States included generic studies for aeromaneuvering and aeroassisted orbital transfer vehicles, ${ }^{2}$ Space Transportation System (STS) support, ${ }^{3}$ defense interceptors design, ${ }^{4}$ Pegasus, ${ }^{5}$ and the National AeroSpace Plane (NASP). ${ }^{6}$ In Europe, programs of note during this same period include HERMES, ${ }^{7}$ Sänger, ${ }^{8}$ and HOTOL ${ }^{9}$ and early work on Huygen ${ }^{10}$ probe. The Buran ${ }^{11}$ development and flight tests were a major focus in the former Soviet Union.

Driving applications for external, continuum, hypersonic flow simulation in the period 1991 to the present included closeouts or continuations of several of the programs noted above as well as $\mathrm{X}-33^{1}$ (a technology demonstrator for a fully Reusable Launch Vehicle (RLV)), X34 (Mach 8 test vehicle), ${ }^{12}$ Assured Crew Return Vehicle (ACRV), ${ }^{13}$ Hyper X, ${ }^{14}$ Commercial Experiment Transporter (COMET) ${ }^{15}$ (later renamed (Multiple Experiment Transporter to Earth Orbit and Return (METEOR)), Mars Exploration Program, ${ }^{16}$ Stardust, Skipper, ${ }^{17}$ and Pegasus XL Wing-Glove Experiment. ${ }^{18}$ Recent advances in computational aerothermodynamics in Europe have been summarized by Kordulla and Morice. ${ }^{19}$ In Japan, OREX and Hyflex with eventual application to the Hope Vehicle dominate hypersonic CFD applications. ${ }^{20-23}$ Though funding is always a concern, there is certainly no shortage of interesting and challenging work to do.

The period prior to 1985 was dominated by significant advances in space marching code capability; predominantly in Parabolized Navier Stokes ${ }^{24}$ work, but also a significantly mature technology base in Viscous Shock-Layer methods. ${ }^{25,26}$ This was also a period in which grid generation and adaption (predominantly structured) addressed challenges associated with flow simulation and resolution around complex vehicles. Hypersonic flows, with the presence of strong shocks and complex interactions presented special challenges that were not adequately accomodated by traditional, body oriented grid systems.

The period from 1985 to 1991 was rich in algorithm advancement and development and/or integration of physical models. Major advances in upwind differencing methods and high resolution, non-oscillatory schemes were introduced in this period. While algorithm and physical model research continue in the period since 1991, these years saw much more emphasis on application of existing algorithms and models to more complex configurations and on ways to exploit computer architectures to accomplish these tasks. Algorithm innovations in this period have been substantially driven by near term needs of programs. The consequences of these shifts have been both good and bad. An underlying theme of this paper addresses these consequences.

\section{Role of Aerothermodynamics in the Entry Vehicle Design Process}

The design of an entry vehicle / reusable launch vehicle such as Shuttle or planetary entry-probe involves the modeling and synthesis of various nonlinear coupled systems. A few important entry vehicle subsystems are propulsion, thermal protection system (TPS), structure/payload, avionics, and cryotanks. The integrated configuration of the necessary subsystems determines the shape of the entry vehicle. The vehicle's shape and the thermal protection system are the components most impacted by aerothermodynamic predictions of the entry flow environment. For a given shape, the aerodynamic performance characteristics of a vehicle (e.g. lift, drag, and static/dynamic stability) are determined using various aerothermodynamic prediction techniques. The TPS, which is the interface between the entry flow environment and the vehicle, is selected, sized, and arc-jet tested based on aerothermodynamic predictions of quantities such as surface temperature, heat transfer rate, integrated heatload, shearloads, pressure loads, and ablation rates.

To better understand the current role of aerothermodynamics in the design process, the process of designing an aeroshell for a propulsively controlled entry probe for landing on the surface of Mars is briefly described. Figure 1 is a process flow diagram for the design of the lander aeroshell; this diagram is representative of the design process used on recent entry probes such as Stardust or Mars/Pathfinder. The diagram shows discipline and subsystem design tasks as a function of time. In general, the fidelity of the analyses 
and the fidelity of the design increases with time. A demarcation between Pre-Phase A and Phase A/B/C is noted in the figure; at this transition in the design process, a large increase occurs in the analysis level of fidelity.

The first step in the design process is determining mission requirements and objectives such as landing a rover at a chosen location and time on the surface of Mars. An initial shape of the vehicle is determined as a function of a number of parameters such as landing accuracy requirements, payload mass and volume, and the launch booster payload shroud. The mass, aerothermodynamic performance of the shape, and the initial conditions as the vehicle enters the Martian atmosphere determine an envelope of possible entry trajectories. The initial aerothermodynamic database is generated using low-fidelity methods such as modifed Newtonian for the aerodynamics and engineering correlations for the stagnation point heating. ${ }^{27}$ Using a 3 degree of freedom (3-DoF) trajectory analysis, an initial design trajectory is generated to meet the mission requirements and satisfy subsystem constraints such as maximum acceleration loads or maximum temperature and heating rates for the TPS.

In the Phase A/B/C segment of the lander design in Fig. 1, the various discipline and subsystem analyses are highly coupled and the design process is iterative. For example, propulsion requirements for control depend on the GN \& $\mathrm{C}$ algorithm; the GN \& $\mathrm{C}$ algorithm depends on knowledge of the lift and drag forces along the trajectory; the lift and drag forces are obtained from the aerothermodynamic predictions. The aerothermodynamic predictions depend on knowledge of the trajectory from the 6-DoF analysis. The 6-DoF analysis is a function of the c.g. location. The c.g. location depends on the packaging and mass properties of the vehicle such as the mass of the payload, TPS, and propulsion system. The mass of TPS and propulsion system depend on the aerothermodynamic predictions. Thus, to determine if the various mission requirements are satisfied, an iterative process is required.

As illustrated above, aerothermodynamics is important in both the early and later stages of the aeroshell design process. In phase A/B/C, accurate predictions of the aerodynamics and entry heating are required to design the TPS and for 6-DoF trajectory analysis. Aerothermodynamic data fully representative of the flight environment are not available from ground based experimental facilities. Thus, high-fidelity numerical simulation techniques, such as Navier-Stokes for the continuum flow regime and Direct Simulation Monte Carlo (DSMC) for the rarefied flow regime, are employed. These methods are computationally intensive and require accurate modeling of relevant physical processes to achieve good results; further, the resources required for a simulation usually increase as the ac-

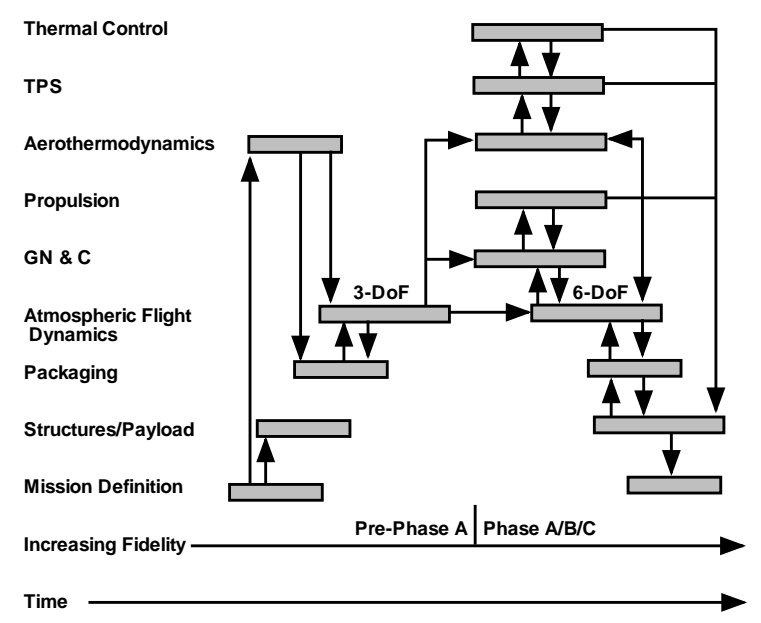

Fig. 1 Design process for a Mars Precision Lander Aeroshell

curacy of the prediction increases. Therefore, to limit costs, high-fidelity aerothermodynamic simulation is only employed in the later stages of the design process after the design has been significantly refined.

The highly coupled and iterative nature of the entry vehicle design process as exemplified by the aeroshell for the Mars lander is a driver for aerothermodynamic development. The process of design involves defining and, then, refining and narrowing the boundaries of a design space until a final solution is reached. To achieve an optimum design, the optimum design solution must always remain within the design space as the design space boundaries are narrowed. Using current low-fidelity, engineering, aerothermodynamic prediction techniques, the preceding design optimization constraint cannot be guaranteed because the engineering methods are not accurate enough. Therefore, to produce optimized entry vehicle designs, new highfidelity aerothermodynamic algorithms and implementation strategies are required that are applicable to the earlier stages of the design process. To achieve this objective, the new algorithms will have to balance accuracy, cost, and speed and take advantage of advanced computational platforms.

\section{Algorithms}

Some of the most important algorithm advances for the computation of hypersonic flows have been in the development of upwind and non-oscillatory schemes. ${ }^{28-30}$ Central difference schemes with upwind modeled or non-oscillatory dissipation operators are included in this algorithm class. ${ }^{31}$ Certainly, upwind schemes evolved more to address issues of temporal accuracy and satisfy an intuitive valuation that consistency between physics and numerics is important. High resolution, non-oscillatory schemes more directly confront issues of accuracy in the near vicin- 
ity of shocks and shear layers. Prior to these advances, flows with strong shocks $\left(p_{2} / p_{1}>\approx 100\right)$ could not be computed accurately with shock-capturing methods; either the requisite dissipation excessively smeared the shock or Gibb's phenomena caused oscillations to negative temperatures on the upstream side of the shock. Shock-fitting methods have advanced to a lesser extent; evolutionary environment has favored the more easily coded and robust shock-capturing methods.

Much algorithm development for hypersonic flows has focused on treatment of source terms. A loosely coupled (split) formulation of the species continuity equations with the main equation set often allows a code developer to retain algorithm structure from a code that does not consider reacting gases. However, fully coupled (unsplit) approaches generally are more robust and converge more quickly than loosely coupled formulations. Within the context of fully-coupled approaches the source term may be handled in a variety of ways to enhance robustness and convergence. In situations where a pure explicit formulation is severely limited by time scales in one or more species source terms, explicit underrelaxation can be selectively applied to species continuity equations. ${ }^{32}$ A more robust approach, easily incorporated in the context of a single step, explicit algorithm, is to utilize point-implicit treatment of the source term. ${ }^{33}$ The Lower-Upper Symmetric-Gauss-Seidel (LU-SGS) ${ }^{34}$ can be modified to achieve good convergence; ${ }^{35}$ though in very energetic situations problems with elemental mass conservation should be addressed. ${ }^{36}$ Convergence rates are generally good on uniform meshes but have been observed to slow on highly stretched grid. All of these formulations sacrifice temporal accuracy for improved convergence with moderate overhead as compared to conventional, explicit formulations.

Convergence acceleration can be addressed on both an algorithmic and procedural level. Examples of algorithmic approaches include various formulations of implicit relaxation, multigrid-methods, defect correction, and preconditioning. Procedural approaches refer to best practice using any available algorithm and include practices such as mesh sequencing and solution sequencing. Most benefit is expected from algorithmic innovation

Implicit relaxation relieves the restriction on the time step associated with highly stretched grids. It also is used to address restriction in time step associated with high Dahmkohler numbers in chemically reacting flows. Examples of implicit formulations for hypersonic flows in chemical nonequilibrium include LU, ${ }^{37,38}$ ADI, ${ }^{38}$ LU-SSOR,$~^{39}$ as well as the LU-SGS methods cited previously. Convergence acceleration of implicit methods for hypersonic, nonequilibrium flows may be achieved using global preconditioning. ${ }^{40} \mathrm{Im}-$ plicit formulations often involve innovations that sacrifice time accuracy for computational efficiency. In such cases, time accuracy may be recovered at the expense of sub-iterations. ${ }^{41}$ In general, temporal accuracy in hypersonic, reacting flow simulations has received only modest attention, and non-trivial issues regarding evolution of systems with disparate time scales (convective, dissipative, multiple reaction groups) may arise. ${ }^{42}$

Multigrid methods have achieved factors of approximately 3.5 reduction in time to convergence for hypersonic flows as compared to application of an otherwise identical algorithm on a single, fine grid. ${ }^{43-45}$ Difficulties arise from treatment of chemical source terms and reduction of high frequency errors in the vicinity of strong shocks. This performance is not as impressive as results obtained in lower speed domains; opportunities for significant advances probably exist.

Local preconditioning in multidimensional flows address problems associated with convergence and truncation error in the limit of incompressible flow. ${ }^{46}$ These problems arise, for example, in the stagnation region of very blunt bodies in hypersonic flow. They are exacerbated in the presence of significant viscous effects. The local Mach number goes to zero in a broad region around the stagnation region and both truncation error and dissipation behave poorly, particularly in upwind schemes. Recent advances suggest that the problems can be alleviated with local preconditioning, but much work remains to establish optimum performance in the vicinity of three-dimensional stagnation points and low Reynolds numbers.

Computational Singular Perturbation (CSP) ${ }^{47}$ exploits concepts that are related to local preconditioning, but focuses on optimal formulation of the chemical kinetic source terms. CSP is of special interest because the method: (1) provides physical insight regarding the balance of kinetic processes in hypersonic reacting flows; and, (2) provides mathematical insight for numerical simulation of such flows to reduce computational expense. Reaction groups are automatically identified which are active, in partial equilibrium, or frozen. Preliminary results were very encouraging; however, coupling the CSP algorithm to the flow solver with requisite modifications to the governing equation set was nontrivial. The concept reached acceptable maturity for uncoupled analyses. ${ }^{48}$

As noted previously, mesh sequencing and solution sequencing are two procedural approaches to convergence acceleration. Mesh sequencing refers to obtaining a converged solution on a sequence of finer grids, where each successive solution is initialized using the previous, coarser grid solution. Solution sequencing refers to initializing a simulation at one trajectory point using a converged solution from a neighboring trajectory point. Solution sequencing can be used in conjunction with mesh sequencing to generate a matrix of solutions across a trajectory for a single configuration. Solution sequencing should not replace mesh sequencing. Based on experience in running ma- 
trices of cases for several X-33 configurations, it is always more efficient to restart a new solution on a coarse mesh initialized with a solution from another trajectory point (solution sequencing) and proceed to sequentially finer grids only after a threshhold residual convergence level has been achieved (mesh sequencing). Proceeding directly to the finest grid (skip mesh sequencing steps) using an initialized solution from another trajectory point generally requires more computer time for a converged solution at the new freestream conditions. The mesh sequencing should be omitted in cases where only a wall boundary condition is changed; here, physically motivated load balancing can be applied to concentrate CPU cycles in the boundary layer.

Similar procedural strategies are applied in block, space marching. ${ }^{49}$ Block marching is a natural extension of the planar, space marching as implemented in Parabolized Navier-Stokes (PNS) solutions. The strategy is useful when (1) embedded subsonic or separated regions may arise in domains which are otherwise amenable to PNS methodology; or (2) the PNS methodology is not sufficiently robust for the given problem. Downstream blocks are initialized using the exit plane data from the upstream blocks. This injection speeds the development of a converged boundary layer profile as compared to conventional, unstaged, global relaxation techniques. Mesh sequencing, from coarse to fine, is still advised in these applications.

\section{Physical Models}

The choice of physical models for an aerothermodynamic flow simulation is a function of the flight environment, TPS, and desired accuracy. The aerothermodynamic flight environment is divided by heat transfer mechanisms. The three primary mechanisms for transferring energy to the surface of a hyper-velocity entry vehicle are: a) energy transfer from particles (atoms, molecules, etc.) colliding with surface, b) energy transfer from chemical reactions on the surface (catalysis), and c) energy transfer via radiation from excited particles in the flow. Mechanisms a) and b) are called convective heating and mechanism c) is called radiative heating.

Accurate aerothermodynamic predictions of convective heating require detailed knowledge of the chemical composition, transport mechanisms, fluid gradients, and TPS material response near the surface of the vehicle. The gas composition near the surface is a function of the path the particles have travelled from the freestream to the shock. Thus, some level of knowledge is required about the flowfield from the freestream through the shock to the surface of the vehicle. To calculate radiative heating, a detailed knowledge of the state of the gas throughout the flow is required. Examples of a few needed quantities are rotational, vibrational, and electronic state populations, coupling

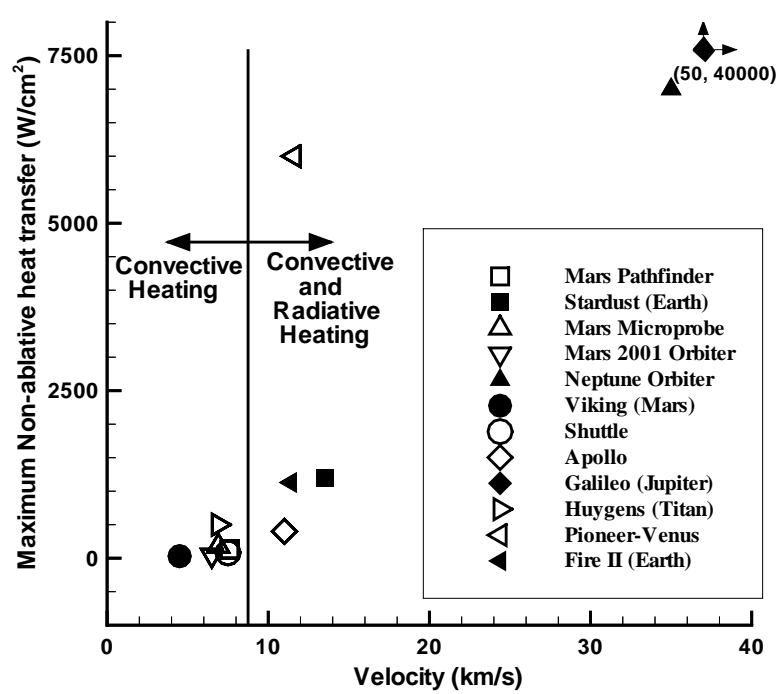

Fig. 2 Peak heating versus velocity for past and future entry vehicles.

and excitation mechanisms between the various energy modes, and modeling parameters for the various molecular and atomic radiation bands. Further, radiation transport at a given location is coupled to all the other locations in a line of sight. In general, radiative heating predictions are much more computationally intensive than predicting convective heating.

The choice of a TPS affects the physical models needed for an aerothermodynamic flow simulation. Ablating and non-ablating materials are two important classes of TPS used for entry vehicle design. Ablating materials are generally employed on nonresuable planetary probes. Ablating materials minimize the energy conducted into an entry probe by utilizing a phase change of the solid TPS material; energy transfer from the flow to the TPS converts the solid TPS material to a gas which is then carried away by the flow. Ablation products injected into the flowfield and surface recession alter the flow environment. Thus, these processes must be modeled to obtain accurate aerothermodynamic predictions. Nonablating TPS is typically used on reusable vehicles such as the Shuttle Orbiter. Nonablating materials minimize the energy conducted into the vehicle by radiating energy away from the body and low material conductivity. For these materials, the flow environment is altered by chemical reactions that occur on the surface of the TPS. These surface catalysis reactions increase surface heating and alter temperature and concentration gradients within the boundary layer. Thus, surface catalysis modeling is needed to accurately predict surface heat transfer.

Finally, the desired level of accuracy is an important consideration in choosing a set of physical models. In general, the computational cost increases as the accuracy of the physical modeling increases. For a 
given vehicle design, the level of accuracy is a function of the available computational resources. Various modeling assumptions such as chemical and thermal equilibrium, frozen flow, tangent slab radiation transport, or a viscous shock layer are made to reduce the cost of a given calculation. These assumptions determine the physical modeling requirements.

Figure 2 is a plot of peak heating rate versus velocity for some past, present and future missions. The peak nonablative heating rates and entry velocities range from $30 \mathrm{~W} / \mathrm{cm}^{2}$ at $4.5 \mathrm{~km} / \mathrm{s}$ for Viking to 40,000 $W / \mathrm{cm}^{2}$ at $50 \mathrm{~km} / \mathrm{s}$ for Galileo. Below $9 \mathrm{~km} / \mathrm{s}$, the primary heating mechanism is convection. At $9 \mathrm{~km} / \mathrm{s}$ and above, the heating is from a mixture of radiation and convection. For Galileo, $99 \%$ of the heating is from radiation. Most of the TPS used for these vehicles is ablative; SLA-561, a silicon based ablator, was used for Viking while carbon-phenolic was used on Pioneer-Venus and Galileo. A recently developed ablator, PICA, is being used for Stardust, a non-ablating Beryllium heatshield was used for Fire II, and nonablating, silica based tiles were used on the Shuttle Orbiter.

\section{Equation of State}

In any hypersonic flow simulation, the thermodynamic pressure is defined as a function of species densities and temperature. The simplest formulation is for a perfect gas $(\mathrm{PG}), p=(\gamma-1) \rho e$. High temperature effects on gas chemistry are sometimes approximated with an effective value of $\gamma$ which yields the correct density ratio across the shock. ${ }^{50,51}$ This approach is useful for aerodynamic analysis if the PG option is the only one available in the code, but is unsatisfactory for evaluation of aeroheating. High temperature effects are more properly handled with chemical equilibrium (CE) or chemical nonequilibrium (CNE) models. In the CE models ( $p=p(\rho, e))$ the functional dependence of pressure on density and energy for a generalized gas mixture (CE-G), in which the elemental mass fractions are known constants or are solved from elemental continuity equations, can be determined by the method of free energy minimization or equilibrium constants. $^{52}$ The method of Liu and Vinokur ${ }^{53}$ can be used to define thermodynamic curve fits for arbitrary gas mixtures based on species partition function information. A very helpful and recent review of partition models for air species has been prepared by Capitelli et. $\mathrm{al}^{54}$ and Giordano et. al. ${ }^{55}$ Curve fits for specific gas mixtures (e.g. air ${ }^{56}$ (CE-air), $\mathrm{CF}_{4}{ }^{57}$ (CE-CF4)) when available are generally less computationally intensive. In the CNE models, pressure is defined as a summation of the partial pressures of each constituent, thermally perfect species $p=\sum_{s} \rho_{s} \bar{R} T / M_{s}$. The partial pressure of free electrons is computed using temperature $T_{e}$ if different from the heavy particle temperature $T$. The species densities are computed from species continuity equations.

\section{Chemical Kinetics}

Chemical source terms may be expressed as functions of thermal equilibrium chemical kinetic models (CNE-TE) or thermal nonequilibrium chemical kinetic models (CNE-TNE). Arhenius formulations for reaction rates are used as the basis for both CNE-TE and CNE-TNE models. Several models for air $^{58}$ and the Martian atmosphere ${ }^{59}$ are available in the literature. Updates to the models have been reported. Some of these updates have been driven by results of the Bow Shock Ultraviolet Experiment in which measured radiative energy transport was in significant disagreement with current theoretical models. ${ }^{60-62}$ In this regard, trace species that are important radiators may be omitted from the initial flowfield simulation and evaluated after the fact, provided their role in important reaction groups and the coupled radiation effects are negligible.

The CNE-TNE models are most often derived from (CNE-TE) models by applying correction factors or effective temperatures to the rate constants. The correction factors for dissociation-recombination are functions of both the vibrational and translation temperatures and may include several additional parameters describing the molecules. ${ }^{63}$ Correction factors for exchange reactions are also available in some models. A very helpful and brief overview of the available models is contained in the recent paper by Losev ${ }^{64}$ (21 models) and Kovach et. al ${ }^{65}$ (15 models). A more comprehensive review (in Russian), associated with the AVOGADRO project, is in the manual by Sergievskaya et. al. ${ }^{66}$

The CNE-TNE models need also address internal energy balance issues. If molecules are preferentially dissociated from higher vibrational energy states, the vibrational energy equation source term must account for this process. The model by $\mathrm{Knab}^{67}$ directly accounts for this relation in the CNE-TNE model. Other models require empirical input.

\section{Thermal State of Species $s$}

The thermal state of species $s$ may be modeled as Equilibrium (TE) or NonEquilibrium (TNE).

The distribution of energy among translational, rotational, vibrational, and electronic modes in species $s$ under equilibrium conditions is defined by the partition function as a function of a single temperature $T$. The specific heat capacity $C_{p}$ of species $s$ is derived from the partition function. Curve fits of specific heat as a function of temperature (TE-C) for species of interest occurring in hypersonic flow simulations (planetary atmospheric constituents, fuels and combustion byproducts, ablation and pyrolisis gases) are available in the literature. ${ }^{68}$ The actual partition functions may also be used (TE-P), particularly if they are already available to support thermal nonequilibrium options 
in the code. In continuum situations, the translational and rotational contributions are generally assumed to be fully excited and the vibrational contributions are modeled as anharmonic oscillators. In many instances, the electronic contribution to the partition function is omitted - only the modes up to and including vibration are retained (TE-Pv). This approximation is probably not bad for conditions when ionization and visible radiation are insignificant; however, we are not aware of studies defining the bounds of this approximation. In any event, the energy does not disappear (assuming conservation laws are formulated correctly!); it is simply distributed among other available modes, thus raising the temperature for a given energy increment.

The TNE models may be subdivided into four subclasses.

The simplest sub-class is the two-temperature model (TNE2) in which it is assumed that the thermal state of species $s$ and all other species in a mixture containing some molecules may be defined by two temperatures, and effects of cross mode coupling are approximated (e.g. rigid dumbell). The translational and rotational energy content of all heavy particles is defined by temperature $T$. The vibrational energy content of all molecules is defined by temperature $T_{V}$ (sometimes called a lumped vibrational temperature model). The electronic and free-electron translational energy modes (if included at all) are usually assumed to be in equilibrium with the vibrational modes at temperature $T_{V}$. (An exception to this approach is discussed by Olynick, ${ }^{69}$ in which electron translational energy modeled as a function of $T$ was found to yield better agreement with experimental data from Project FIRE.) Justification for this last assumption in air is based on efficient energy transfer between electrons and the vibrational modes of molecular nitrogen. The TNE2 model may be implemented in two ways. If equilibrium curve fits are available, as discussed previously, the vibrational and electronic heat capacity of species $s$ is easily defined as a function of $T_{V}$. It is obtained by subtracting the simple translational-rotational contribution from the curve fit for all energy modes appropriate for each atom or molecule (TNE2-C). If partition functions are available, the appropriate temperature is substituted into the function for each energy mode (TNE2-P). As before, if only the modes up to and including vibration are retained, the model is designated (TNE2-Pv).

The next logical TNE sub-class is the threetemperature model (TNE3). It is identical to the TNE2 sub-class except that the electronic and freeelectron translational energy partition is defined by a third temperature $T_{e}$. (This designation is a misnomer if there are no molecules present in the gas.) This model has been considered primarily for high speed Earth entries characteristic of return from geosynchronous Earth orbit or above. If molecules are present, the use of curve fits is not possible because the relative contributions of vibrational and electronic modes to the energy content are not separable without some partition function information.

The third TNE sub-class is the multi-temperature model (TNEM). It is identical to the TNE2 sub-class except that each molecular species $s$ retains it own vibrational temperature $T_{v, s}$. When the thermal model retains only the modes up to and including vibration, the model is designated (TNEM-v). Such models are most often used in the code validation process using ground-based experimental data in which separate vibrational temperatures have been measured. Separate rotational temperatures may also be considered in this classification. Such an approach is featured in a paper by Bose et. al. ${ }^{60}$ in which the high altitude kinetics required for accurate prediction of NO number densities and radiation for the Bow-Shock Ultraviolet experiment necessitated more complete characterization of the molecular energy content in the kinetic model. ${ }^{70}$ Separate electronic temperatures $T_{e, s}$ logically follow in this model, but we are unaware of studies involving (or justifying) this degree of difficulty.

All of the TNE models to this point invoke an assumption of equilibrium partitioning of energy within two or more groupings of internal modes. This assumption can be relaxed further by discretizing each species $s$ into multiple sub-species (excited states) as a function of vibrational and/or electronic quantum number(s). ${ }^{71}$ In this fourth TNE sub-class, a statespecific approach (TNEQ), a unique translational temperature is still appropriate within the context of a continuum flow simulation; however, the role played by modal temperatures in defining energy distribution within the mode is replaced by a discrete accounting of various excited states. Each state requires its own continuity equation, with appropriate models for transport and source terms.

\section{Transport Properties and Diffusion Models}

Transport properties are required to describe the diffusion of mass, momentum, and energy in a gas. A comprehensive review of the subject is provided in the text by Hirschfelder, Curtiss, and Bird. ${ }^{72}$ The simplest definition, usually restricted to perfect gas simulations, employs Sutherlands law for viscosity, $\mu=c_{1} T^{3 / 2} /\left(c_{2}+T\right)$, and Prandtl number for conductivity, $k=\mu C_{p} / \operatorname{Pr}$, where $c_{1}, c_{2}$, and $\operatorname{Pr}$ are constants. Thermochemical equilibrium flow simulations most often use curve fits for $\mu$ and $k$ as a function of temperature and pressure. ${ }^{73}$ Transport properties in thermochemical nonequilibrium flows are defined as functions of constituent species transport properties and respective mole fractions in the mixture. Individual species transport properties are defined as functions of collision cross sections; often this data is readily available in form of curve fits as functions of temperature. ${ }^{74}$ Mixing laws for viscosity and thermal 
conductivities based on work by Yos $^{75}$ and Wilke ${ }^{76}$ are also discussed and compared to a simple, mole fraction weighted average by Palmer. ${ }^{77}$

The definition of diffusion coefficients should be discussed within the context of the diffusion model. Full multicomponent diffusion involves expensive computation of multicomponent diffusion coefficients as a function of binary diffusion coefficients. Application of the Stefan-Maxwell relations simplifies the requisite formulation of diffusion coefficients, but still involves significant overhead (e.g. evaluation of binary diffusion coefficients and sub-iterations) in the computation of multicomponent diffusion. These approaches are equivalent in terms of simulation accuracy and they rigorously conserve mass; the sum of all species continuity equations telescopes (term by term cancellation) exactly to the mixture continuity equation. A simplification, originally derived and justified for trace species, ${ }^{78}$ involves formulation of an effective binary diffusion coefficient $\left(\mathrm{D}_{s, m}\right)$ for species $s$ using reciprocal molal averages of binary diffusion coefficients $\left(D_{s, k}\right)$, where the mass diffusion flux $j_{s}$ is driven by the gradient of mole fraction of species $s\left(X_{s}\right)$ in the absence of pressure and thermal diffusion. This formulation is expressed

$$
\tilde{j}_{s}=-\rho \frac{M_{s}}{M}\left(1-\omega_{s}\right) \mathrm{D}_{s, m} \nabla X_{s}
$$

where

$$
\frac{1}{\mathrm{D}_{s, m}}=\sum_{k \neq s} \frac{X_{k}}{D_{s, k}}
$$

$\rho$ is density, $\omega_{s}$ is mass fraction of species $s$, and $M$ and $M_{s}$ are mixture and species molecular weights, respectively. In applications involving velocities below 7 $\mathrm{km} / \mathrm{s}$ in air on the Space Shuttle, the heating component associated with the diffusion of atoms toward the surface and their catalytic recombination is adequately described by this approach. ${ }^{49}$ However, species continuity equations written in this simplified manner do not generally telescope into the mixture continuity equation because the approximation to diffusive mass flux $\tilde{j}_{s}$ to not telescope to zero. At higher velocities and/or at smaller length scales where nonequilibrium effects associated with the catalytic recombination become more pronounced, the approximation to multicomponent diffusion will deteriorate.

Three approaches may be applied to correct this deficiency in mass conservation when approximating multicomponent diffusion of species $s$ as a function of a single gradient in species $s$. The bifurcation approximation closely approximates actual diffusion coefficient data in a manner that imposes mass conservation. ${ }^{79}$ Explicit corrections on the diffusion fluxes may be imposed that remove a net background flux in Eq. 1.

$$
j_{s}=\tilde{j}_{s}-\omega_{s} \sum_{k} \tilde{j}_{k}
$$

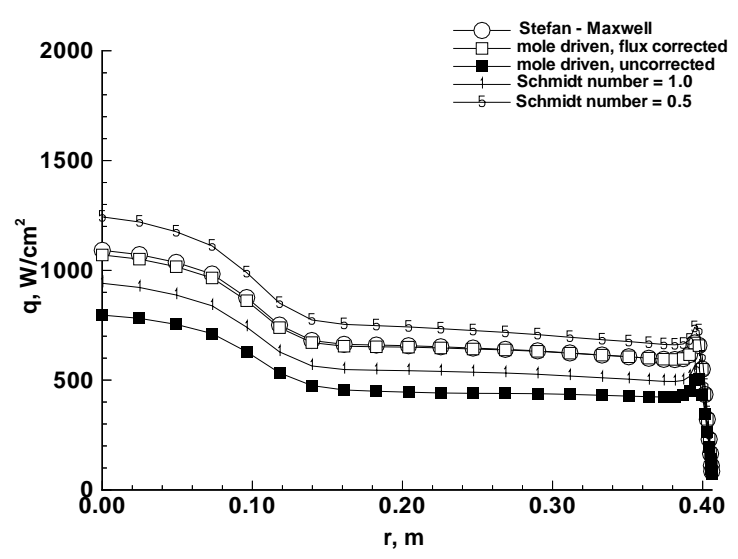

a) $V_{\infty}=10.9 \mathrm{~km} / \mathrm{s}$

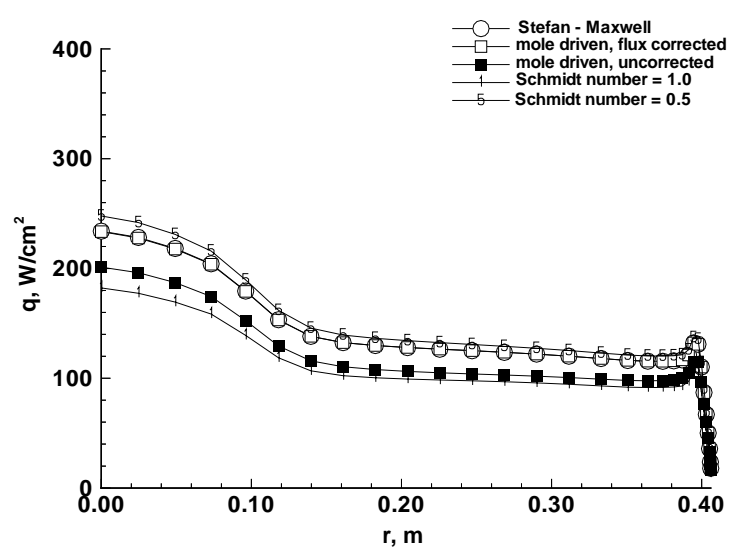

b) $V_{\infty}=7.0 \mathrm{~km} / \mathrm{s}$

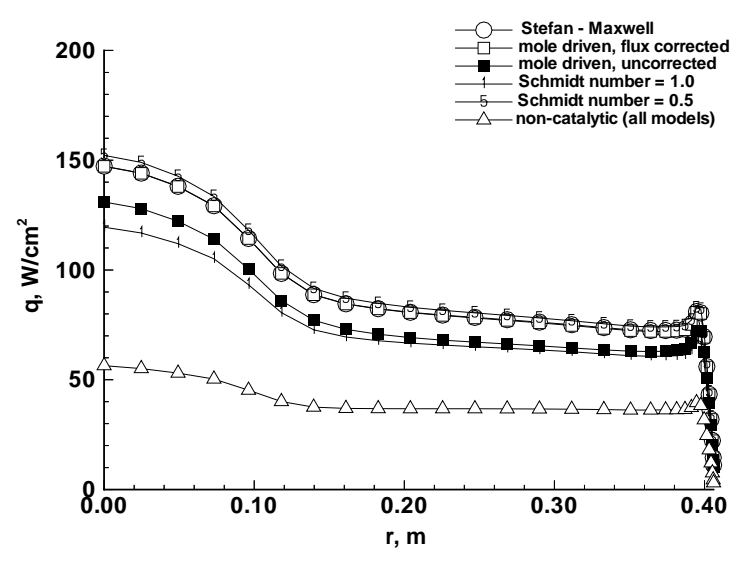

c) $V_{\infty}=6.0 \mathrm{~km} / \mathrm{s}$

Fig. 3 Convective heating distribution on Stardust vehicle with various approximations to multicomponent diffusion.

Finally, all diffusion fluxes may be reformulated as a function of viscosity $\mu$ and a constant Schmidt number $S c$.

$$
j_{s}=\frac{\mu}{S c} \nabla \omega_{s}
$$


The effects of these approximations are seen in Fig. 3 in which the convective heating to the Stardust vehicle are computed at an actual trajectory point $\left(V_{\infty}=10.9\right.$ $\mathrm{km} / \mathrm{s}, \rho=2.6910^{-4} \mathrm{~kg} / \mathrm{m}^{3}$ ) and at progressively lower velocities at the same density. The mole driven, uncorrected results refer to Eq. 1. The mole driven, flux corrected results refer to Eq. 3. At all energies, the flux corrected result is in excellent agreement with the Stefan-Maxwell relations. As flow energy decreases the uncorrected approximation approaches the StefanMaxwell reference, with average error decreasing from $30 \%$ at the highest velocity, $15 \%$ at $7 \mathrm{~km} / \mathrm{s}$, and $12 \%$ at $6 \mathrm{~km} / \mathrm{s}$. Even at $6 \mathrm{~km} / \mathrm{s}$ (Mach 19) the contribution to total heat flux from diffusion of species to the surface under fully catalytic conditions is quite large as compared to the non-catalytic surface case. The heating for all approximations to multicomponent diffusion are nearly identical when the diffusional contribution is removed at a non-catalytic wall. The effective Schmidt number changes across the velocity domain tested here, with $\mathrm{Sc}=0.5$ a reasonable approximation at the lower velocities.

\section{Surface Catalysis and Ablation}

In a hypersonic flow, surface catalysis is a primary mechanism for transferring energy to the surface of an entry vehicle. Catalytic surface heat transfer occurs when the TPS material at the fluid-surface interface acts as a catalyst to an exothermic reaction involving chemical species impinging the surface from the flow. A fraction of the catalytic energy released at the surface is conducted into the vehicle; the additional heating increases the TPS mass. Atomic oxygen combining to form $\mathrm{O}_{2}$ or atomic nitrogen combining to form $\mathrm{N}_{2}$ are important surface catalytic reactions in air. $\mathrm{CO}$ combining with $\mathrm{O}$ to form $\mathrm{CO}_{2}$ is an important catalytic reaction in an atmosphere with a large $\mathrm{CO}_{2}$ component such as Mars.

Surface catalysis is an especially important heating mechanism for dissociated flows in chemical nonequilibrium. In general, the surface of a hypervelocity entry vehicle is much cooler than the flow temperature directly behind the shock. For a boundary layer in chemical equilibrium, most of the dissociated species recombine before they reach the surface. Therefore, the majority of surface heat transfer is caused by particle collisions with the surface (convection). For a flow in chemical nonequilibrium, flow species which dissociate in the shock-layer do not have time to completely recombine (three-body collision required for recombination) before impinging the vehicle's surface. Thus, the catalytic fraction of the total heat transfer rate is significant and must be considered to accurately predict the surface heating.

For the Shuttle Orbiter, catalytic heating is important because the Shuttle entry trajectory envelope is bounded by TPS material temperature limits on the nose and leading edges where the magnitude of catalytic heating is largest; ${ }^{80}$ For RLV, this will also be true. Thus, the ability to predict catalytic heat transfer is directly linked to Shuttle entry performance. Predicted magnitudes for catalytic heating over the Shuttle surface are as follows. ${ }^{81}$ Near the nose, the catalytic fraction of the heatload is $15-40 \%$ of the total and increases as the stagnation point is approached. On the wing leading edges, the catalytic fraction is between 10-20\%. On most of the windside acreage, the catalytic fraction is between $5-10 \%$. Finally, on most of the leeside surfaces, the catalytic fraction is negligible.

For most CFD applications, the modeling of surface catalysis is based on simple kinetic theory. For atoms impacting a surface and recombining, the surface reaction rate is assumed proportional to the number flux of atoms striking the surface times a catalytic efficiency; the catalytic efficiency is the fraction of atoms that recombine on the surface. These atoms are supplied by diffusion. Thus, in terms of the mass flux of species $s$, a catalytic surface boundary condition is given by, ${ }^{82}$

$$
\left(j_{s}\right)_{w}=\rho_{s w} \gamma_{s} \sqrt{\frac{k T_{w}}{2 \pi m_{s}}}
$$

where $\gamma_{s}$ is the catalytic efficiency. A more rigorous approach to surface catalysis would involve modeling such phenomena as the availability of surface reaction sites, the rate at which surface reaction sites are filled or depleted, surface energy accommodation, and actual surface reaction rates.

For a number of resuable TPS materials and coatings, $\gamma$ curve-fits for atomic oxygen and nitrogen surface catalysis have been experimentally determined as a function of temperature ${ }^{83}$ as,

$$
\gamma_{s}=a_{s} \exp \left(-\frac{b_{s}}{T_{w}}\right)
$$

Details of the experimental procedure which involves arc-jet testing are described by Stewart. ${ }^{84}$

A fully catalytic wall boundary condition is specified in Eq. 5 when $\gamma_{s}=1$ (i.e., all atoms striking the surface recombine). A non-catalytic wall is specified when $\gamma_{s}=0$ (i.e., the surface does not catalyze reactions). Depending on the material and the flow environment, the ratio of heating between a fully catalytic wall and a non-catalytic wall is as high as 2 or 3 .

All of the discussion on surface catalysis has assumed a reusable, nonablating TPS system. The physics and models for ablating and charring systems has recently been reviewed by Milos and Rasky ${ }^{85}$ and Milos and Chen. ${ }^{86}$ Significant, additional complexity can be introduced in these situations because of the additional chemical species introduced into the flow and boundary conditions dependent on a coupled material response. Simpler, uncoupled analyses may also 
be accomplished in which species mass fractions and temperatures at a surface are supplied to the flowfield analysis from an external source.

\section{Turbulence and Transition}

Algebraic turbulence models (e.g. BaldwinLomax, ${ }^{87}$ Cebeci-Smith ${ }^{88}$ ) remain the favored approach to turbulence modeling in hypersonic flowfield codes. Use of local values of shear in the damping function formulation are generally required in these methods in hypersonic applications. ${ }^{89}$ Provisions for separation, as in front of deflected bodyflaps, can be accomodated in some situations. ${ }^{90}$ However, such models are not generally applicable in regions of massive separation as occur on the leeside of vehicles at high angle of attack or in the wake, in corners, or in gaps. The algebraic models will often produce converged solutions in regions where the model is inappropriate; the user must carefully interpret such results.

One- and two-equation turbulence models ${ }^{91}$ are more generally applicable. As experience and confidence increases with these models, they are replacing the algebraic models for flowfield simulations over complex configurations. Several models used in hypersonic airbreathing propulsion applications in the LARCK code have been cited recently by Kumar et. al. ${ }^{92}$

Modeling / prediction of transition fronts has taken different tacks in applications associated with hypersonic airbreathing propulsion and rocket propelled vehicles. Prediction of transition is a far more critical issue in the case of hypersonic airbreathing propulsion vehicles because drag due to skin friction can severely limit performance, yet turbulent mixing of hydrogen and air in the combustor is required. State-of-the-art methods, recently reviewed in several sources, ${ }^{93,94}$ are being developed and tested with these applications in mind. ${ }^{95}$ The status of flight data to evaluate such models was recently discussed by Bushnell. ${ }^{96}$

The approach to transition prediction can afford to be somewhat more conservative in rocket powered vehicles (e.g. Reusable Launch Vehicle, X-33, X-34, $\mathrm{X}-38$ ). The effect of transition on aerodynamics is less significant on these blunter bodies at high angle of attack. Also, peak turbulent heating levels are usually less than peak laminar heating levels on return from low-Earth-orbit. (This is not necessarily the case in suborbital test flights.)

Wind tunnel data from conventional (non-quiet) tunnels are believed to provide conservative estimates for transition behavior except for cases in which extreme roughness or trips emerge on the flight hardware. Flight experience from Space Shuttle provides additional information on the range of conditions at which transition may occur on tiled surfaces and some instances where obstructions inadvertantly introduced between tile gaps caused early transition. ${ }^{97}$ It has been observed that transition fronts in the wind tunnel for $\mathrm{X}-33$ are not necessarily coincident with contours of momentum thickness Reynolds number. However, momentum thickness Reynolds number appears to be a reasonable metric for defining the first occurrence of transition on the vehicle in the wind tunnel. The magnitude of this metric for transition prediction is a function of roughness height to boundary-layer thickness. Transition fronts in computation of X-33 are then introduced across a computational coordinate or zone boundary based on wind-tunnel derived metrics.

\section{Radiation Coupling}

A few mission scenarios where radiative heating is important for entry capsule design are sample returns, outer planet, and manned Moon or Mars missions; all of the mission types employ high-velocity direct entry trajectories for landing. Another area where radiative heating is important is rocket base heating via plume radiation on ascent trajectories. As an example, six missions are in various stages of development involving sample returns to Earth: Stardust, Genesis, Alladin, Mars Sample Return, Champollion, and Muses-C; these missions return samples from the tail of comet, solar wind, the moons of Mars, the surface of Mars, the surface of a comet, and the surface of an asteroid, respectively. Earth entry velocities for these missions vary from $12-16 \mathrm{~km} / \mathrm{s}$. For Earth entry, radiation as a heating mechanism becomes significant for atmospheric entry velocities above $10 \mathrm{~km} / \mathrm{s}^{98}$ Thus, radiative heating will be important for all of these missions.

Computationally, radiation coupling is important when the fraction of flowfield energy consumed by radiation excitation is significant; the amount of radiation is overpredicted when radiation coupling effects are neglected. The radiation is overestimated because radiation coupling removes energy from the flow. If this energy sink is not considered in the simulation, then the excess energy goes toward chemical and internal energy mode excitation which increases the amount of predicted radiation. This situation is analogous to chemical or internal energy coupling in a flow simulation. As an example, if a perfect gas formulation is used to model a high-velocity entry flow, then the post shock temperature is grossly over-predicted. Further, if the perfect gas temperature is used to calculate the chemical composition of the gas, then the amount of ionization and dissociation will be overestimated.

The amount of radiation in a flow field is a function of the geometry, atmosphere, and entry trajectory. Physically, the amount of radiative heating is proportional to the shock-layer thickness which increases as the nose radius increases. Conversely, convective heating decreases as the nose radius increases; the magnitude of the radiative heating at the stagnation point is proportional to the nose radius while the con- 
vective heating is inversely proportional to the square root of the nose radius. ${ }^{99}$ For example, Stardust (12.6 $\mathrm{km} / \mathrm{s}$ relative Earth entry velocity, $8.2^{\circ}$ entry angle, $60^{\circ}$ sphere-cone, nose radius of $.23 \mathrm{~m}$, maximum diameter of $.8 \mathrm{~m}$ ) has a peak total non-ablative heat transfer rate of $1300 \mathrm{~W} / \mathrm{cm}^{2}$ where the radiative component is about $10 \%$ of the total. Fire $\mathrm{II}^{100}(11.4 \mathrm{~km} / \mathrm{s}$ relative Earth entry velocity, $14.6^{\circ}$ entry angle, nose radius of $.8 \mathrm{~m}$, maximum diameter of $.63 \mathrm{~m}$ ), a flight experiment in early 1960's, has a peak total non-ablative heat transfer of $1140 \mathrm{~W} / \mathrm{cm}^{2}$ where the radiative component is about $40 \%$ of the total. Thus, because the Fire II vehicle has a larger nose radius and a steeper entry trajectory, the magnitude of the radiative heating and its fraction of the total is much larger for Fire II than for Stardust despite the lower entry velocity for the Fire II vehicle.

For manned missions, vehicle dimensions are usually much larger than for the robotic missions. Therefore, convective heating is reduced, but the amount of radiative heating is increased. For example, on Apollo $\left(11 \mathrm{~km} / \mathrm{s}\right.$ entry), peak heating is about $350 \mathrm{~W} / \mathrm{cm}^{2}$ with half the total coming from radiation.

Finally, the composition of the atmosphere affects the magnitude of radiative heating and its spectral distribution. For example, for an equivalent trajectory and geometry, radiative heating in air is less than radiative heating in a $\mathrm{CO}_{2}-\mathrm{N}_{2}$ mixture representative of the Martian or Venusian atmospheres. For $\mathrm{H}_{2}-\mathrm{H}_{e}$ mixture representative of the Jovian atmosphere, an entry velocity on the order of $35 \mathrm{~km} / \mathrm{s}$ is needed to generate the post shock temperatures for air at $11 \mathrm{~km} / \mathrm{s}$. Thus, for outer planet missions, the entry velocity at which radiative heating becomes important is much higher than for an Earth entry.

Computationally, the high-fidelity simulation of a radiating flowfield is daunting; radiation modeling can increase the cost of a calculation by orders of magnitude. At the high entry velocities where radiation is important, flow species are both dissociated and ionized. Thus, for a Navier-Stokes simulation with finite rate chemistry and thermal nonequilibrium, a large number of physical processes are modeled and a stiff equation set must be solved; this requirement is true regardless of whether radiation effects are included. With radiation, the number of physical processes that are modeled is orders of magnitude greater. Further, ablative TPS materials are typically used for vehicles with large entry velocities. The inclusion of ablation products adds more complexity because they can absorb radiation. Thus, the radiation properties of the ablation products must be modeled to accurately predict the surface radiative heating.

Radiation intensity predictions over a relevant spectral range at a point in an entry flowfield requires emission and absorption coefficients for important radiation mechanisms, excited state population distri- butions, and radiation transport. To calculate the absorption and emission coefficients, a large database of modeling parameters is required; these parameters are estimated from experimental data and theoretical formulations which are sometimes questionable for the velocity range or atmosphere of interest in entry capsule design. To predict radiative intensities, excited state population distributions are needed; radiation intensity is directly proportional to the number of particles in an excited state. For typical gas mixtures, there are thousands of excited states. If the excited state populations are in nonequilibrium, then without simplifying approximations, a rate equation is needed for each state. Again, this requires a vast database of modeling parameters. Finally, the radiation transport at thousands of spectral frequencies must be calculated; radiation is attenuated through emission and absorption as it travels from one point in the flow to another. Therefore, the radiation intensity at each point in the flow is a function of every other point in the flow within a line of sight; the radiation transport algorithm models this effect.

In recent years, strides have been taken to improve flowfield radiation prediction and coupling techniques. For modeling nonequilibrium radiation, the NEQUAIR $^{101}$ and LORAN ${ }^{102}$ codes were developed. NOVAR, a derivative of LORAN, has been loosely coupled with the flow solver GIANTS to generate NavierStokes solutions with coupled radiation for comparison with the FIRE II flight data. ${ }^{69}$ Using a tangent slab radiation transport algorithm, a good agreement with the flight data (total stagnation heating and radiative intensity) over the entry trajectory was obtained; further, radiative coupling lead to a better comparison with the flight data as compared to an uncoupled simulation. Most calculations with radiation coupling have used a tangent-slab approximation to model the radiation transport; the assumptions of this approximation are valid in the stagnation region of a blunt body flow. Using LAURA and LORAN, however, a few Navier-Stokes calculations have been generated with coupled radiation and a multi-dimensional radiation transport algorithm. ${ }^{103}$ Moving to higher levels of approximation, flow predictions with radiation coupling have been performed, as non-equilibrium and 1- $\mathrm{D}^{104}$ and VSL with equilibrium radiation and ablation. ${ }^{105}$ For Hydrogen-Helium, radiation work done in the late 1960 's would still be considered state-of-the art. ${ }^{106}$

The accurate modeling of radiation and radiation coupling is one of the great remaining challenges in computational aerothermodynamic design. With the success of Galileo, our ability to design an entry capsule and TPS to survive tremendous heatloads dominated by radiative heating was confirmed. The heatshield, however, was over-designed and the pre-flight predictions were not very accurate based on current interpretation of flight data. ${ }^{107}$ Even in cases where 
the magnitude of radiation is low, recent flight experiments reveal serious shortcomings in some elements of the models. ${ }^{61,62}$ A question that remains to be answered is: for flows with significant radiation coupling, can we develop prediction methodologies with enough accuracy to reduce design margins? To achieve this goal, research and development is needed to couple radiation and ablation to 3-D Navier-Stokes solvers, develop appropriate radiation process models (particularly for other planetary atmospheres, develop multidimensional radiation transport algorithms, and continue to improve nonequilibrium radiation modeling.

\section{Low Density Flows}

Computation of nozzle flows and wake flows often involve severe expansions into domains that are not adequately modeled with the continuum approximations in the Navier-Stokes Equations. Bird ${ }^{108}$ (Eq. 8.45) defines a breakdown criteria of translational equilibrium in expansions based on physical arguments. Numerical error, particularly that associated with approximate Riemann solvers in these extremes, may cause the continuum solutions to deteriorate even earlier as manifested by entropy production. ${ }^{109}$

Similar problems occur in continuum simulations of hypersonic flows over vehicles at high altitude. Direct Simulation Monte Carlo methods offer the best simulation capabilities at these rarefied conditions. Application of models for temperature, concentration, velocity, and pressure slip ${ }^{110}$ in a continuum simulation extend the altitude range for stagnation point heating agreement with DSMC to $115 \mathrm{~km}$ for the Shuttle Orbiter nose radius at $7.5 \mathrm{~km} / \mathrm{s} .{ }^{111}$ Pitching moment coefficient prediction by DSMC and NavierStokes simulations for the COMET reentry vehicle at $90 \mathrm{~km}$ and $60 \mathrm{deg}$ angle of attack are also in very good agreement. ${ }^{112}$ However, significant disagreement in shock thickness and shock layer profiles exist down to at least $90 \mathrm{~km}$ in these cases.

\section{Surface Definition and Grid Generation Structured Grids}

Grids are the foundation upon which all hypersonic CFD analysis is built and without quality grids, it is impossible to obtain solutions of high accuracy. The grid generation process represents one of the greatest impediments to the timely application of CFD to the design process. At the present, the creation of a high quality CFD grid is a labor intensive process requiring skilled workers with access to only a few automated procedures and requiring days or weeks to complete. Although grid generation software has improved greatly in the past decade, it is still an "art" to create a quality grid.

\section{Surface Modeling}

All complex surfaces are generated on any number of commercial or company proprietary CAD/CAM systems which theoretically should be able to communicate through the standard IGES protocol with other design systems as well as grid generation software. Experience has shown that this communication between CAD/CAM systems and grid generation software all to often is less than adequate. To alleviate this problem, NASA created the NASA IGES ${ }^{113}$ software that takes any generic IGES file and creates a file compatible with grid generation software used by NASA. This step has improved the communication process but there is an additional disconnect between designers and grid generators. In general, designers using CAD/CAM are not sensitive to the surface quality requirements for grid generation. As a result, considerable time can be required to "repair" a surface representation before the grid generation process can begin. The best results are obtained when grid generators become involved early in the design process so their requirements can be incorporated in the surface model.

\section{Surface Grids}

At the present time, the surface grid generation process is the most time consuming and labor intensive element in grid generation. It is at this point that the "patches" or surfaces, which can number in hundreds for complex configurations, must be pulled together, grid densities and distributions imposed on the grid, grid topology established, the grid smoothed and projected to the surface. There are a number of commercial, public domain and company proprietary software packages such as ICEM, ${ }^{114}$ GRIDGEN, ${ }^{115}$ NGP,${ }^{116}$ GENIE $^{117}$ and EAGLE ${ }^{118}$ to name a few that can be used to generate surface grids and block faces of a computational domain. Most of these codes include a graphical interface to enhance the process.

Parametric studies, i.e. control surface deflection, body shape change, etc., can require only minor changes in or complete rebuilds of the surface grid depending on the nature of the grid. Several of the codes mentioned above offer the option to build a parametric model of the configuration and the automation of parametric changes in the grid at a high cost in initial set up time. This approach is effective when the set up costs can be spread out over a long term project but not applicable to short term "paper studies" or when an evolving vehicle is undergoing large and frequent geometric changes.

\section{Volume Grids}

Volume grid generation is the most automated element of the grid generation process. This is the point at which grid boundary conditions are set and the grid can be smoothed. This process is normally accomplished in a batch mode on either a workstation or mainframe computer depending on the size of the job. The ICEM, NGP, GENIE, and EAGLE codes all 
have a volume grid generation capability. In addition, GRIDGEN3D, ${ }^{115}$ 3DGRAPE, ${ }^{119}$ 3DMAGGS ${ }^{120}$ and 3DGRAPEAL ${ }^{121}$ are stand alone elliptic grid generators. Also Alter ${ }^{122}$ has developed software that can be used to quickly make local alterations in existing volume grids. Resolution of important flow features which are not necessarily aligned with the surface or bow shock can be accommodated using solution adaptive methods as represented by SAGEv2. ${ }^{123}$

\section{Unstructured Grids}

While subsonic and transonic viscous analyses have been demonstrated with some success using unstructured grids, such efforts for hypersonic flows with heat transfer are less evident. ${ }^{124}$ There is, however, a maturing technology base for application of unstructured grids to inviscid, hypersonic flow analyses.

Although unstructured grid generation suffers from the same surface definition problems encountered in structured grid generation, once the initial set up is done the construction of subsequent surface and volume grids is quick and efficient - on the order of hours - which makes this approach attractive for parametric analysis. There are two basic types of unstructured grids, the traditional based on tetrahedra and cartesian based on hexahedra.

\section{Surface Grids}

Like the structured grid process, connectivity must be established between surface patches and grid distributions specified before the surface grid can be generated. Most software has an interactive front end to aid in the process. In general, this element of the process is much less labor intensive and more automated relative to structured grid generation. The FELISA ${ }^{125}$ and VGRID $^{126}$ codes represent mature software for traditional unstructured grid generation and the algorithm described by Aftosmis et. al. ${ }^{127}$ is representative of unstructured cartesian software.

\section{Volume Grids}

Once the boundaries of the computational domain are established and parameters are set to control grid spacing, the grid is created in a batch mode. FELISA, VGRID and unstructured Cartesian solvers all possess self-consistent volume grid generators.

\section{Hardware Requiremments}

Except for the most complex grids, a high end workstation with 750 Mbytes of memory is sufficient for structured and unstructured grid generation. For example, a 127 block volume grid having a total of 5 million grid points was generated on a Silicon Graphics /R10000 machine with 750 Mbytes of memory.

\section{Validation and Error Estimation \\ General Comments}

Validation and error estimation are critical challenges within computational aerothermodynamics because uncertainties in predicting vehicle aerothermodynamic performance increase design margins; large design margins add weight and lower vehicle performance. ${ }^{128}$ Validation requires the user check that (1) the governing equations are being solved correctly and (2) the correct and sufficient set of equations (physical models) are being solved. The first set of checks involve things like: temporal and/or spacial grid convergence studies; conservation checks on elemental mass fractions, entropy, and total enthalpy; comparisons to known analytic solutions for simple, well-posed problems; independent recoding of program modules; and comparisons to experimental data with insignificant uncertainty in physical models. This aspect of validation has much in common with other flow domains, has been described in the literature, ${ }^{129}$ and will not be elaborated on here. The second set of checks predominantly involve comparisons to experimental data in which effects of empirically or approximately modeled physical phenomena are manifest in the measurements. The requisite physical properties and models for computational aerothermodynamics have been discussed in a previous section. Uncertainties in these physical properties and models tend to escalate with increasing energy of the flow. The difficulties and cost of obtaining experimental data for code validation also escalate with increasing energy of the flow.

Error estimation is predominantly based on code validation experience. In any given design application, the code is validated against experimental data sets that most closely match configuration and flight parameter space (Mach number, Reynolds number, total enthalpy and pressure, wall temperature, surface roughness, etc.). Under the most ideal circumstances, error estimation is based on fully grid converged solutions in which the application parameter space is fully contained within a validation test set parameter space. These ideal circumstances are often attainable in CFD applications ranging from incompressible to supersonic. At hypersonic conditions, extrapolation of error estimate beyond any available validation set parameter space is usually required.

Grid convergence studies are a necessary but insufficient metric for establishing error estimates in the less-than-ideal circumstances that usually prevail in hypersonic applications. In geometrically simple but physically challenging validation experiments grid converged solutions may be realized and attention can be focused on the physics. Experimental programs ${ }^{130,131}$ and workshops have been initiated to address these component focused needs while trying to minimize the possibility that coupled modeling errors may fortuitously cancel. Even with simple shapes, such studies 
are not trivial when the entry velocities (energies) are sufficiently high (e.g. Galileo, Stardust, Apollo) that radiation and ablation must be included in the simulation.

In more geometrically complex configurations, obtaining a grid converged solution is either not possible or is precluded by higher priority claims on computer resources. (This situation is changing with advances in workstation clusters and validation data sets like the STS OEX data will need to be revisited.) In these instances the validation data set used to establish the uncertainty in computed heating levels or aerodynamics should be defined along with an appropriate description of the relevant parameter space and level of grid convergence. The sensitivity of the result to model parameters can be defined. Evaluation of the relative contributions to heating rate (thermal conduction, diffusion, radiation) or aerodynamic forces (pressure, skin friction, base pressure) can sometimes provide insight into the error estimation process.

Ultimately, confidence in CFD predictions depends on comprehensive comparisons to experimental data with well defined error bounds. The greater the inventory of validation sets, the better one is able to deal with complexity and to make error estimates. Consider, for example, the large flight aerothermal database that exists for the Shuttle Orbiter. At what point in a series of validation tests using this data is a code validated for aerodynamics and heating predictions? Certainly a single test case, even one with a single grid refinement that produces changes in aerodynamics and heating less than experimental uncertainty, is only a starting point. Isolating physical modeling errors from discretization errors in a single test case is difficult because of the complexity, size, and large number of coupled physical processes needed to simulate the flowfield around the Shuttle Orbiter. Discretization and physical modeling errors are coupled because the physical modeling is a function of flow parameters (velocity, temperature, pressure, etc.) and their gradients which are a function of grid resolution. Further, many of the physical processes considered such as vibrational excitation and dissociation are coupled. Thus, errors from one physical model propogate to other models and isolating errors is difficult; good agreement with experiment may simply represent fortuitous cancelling of errors.

Taking the question a step further: Are consistent comparisons with experimental data along multiple points along a single trajectory sufficient for validation? Are multiple points along several different flight trajectories sufficient? The multiple tests now begin to provide a statistically meaningful basis for error estimation for a particular configuration and parameter space. Fortuitously cancelling errors are less likely though still possible.

What happens if a new configuration is proposed in which bodyflap deflections are larger than the Shuttle Orbiter at hypersonic, reacting gas conditions? If one has high enthalpy wedge experiments in the validation inventory, then reasonable error estimates may be derived. (Note that data sets for wedge experiments may need to account for three-dimensional effects. Also, some recent experimental data has suggested more uncertainty in established models for what had been thought to be a "simple" problem. ${ }^{132}$ )

Code validation is a process. The process continues until no statistically significant reduction of error estimate can be realized for a specific application. Even in the limit of infinitely fine grid, the error estimate cannot go to zero if experimental measurements are required to anchor physical models within the simulation. Simulation uncertainties depend on the quantity being predicted (aerodynamics, heating rate, electron number density) and increase with the complexity of the flow. The complexity of the flow is a function of both geometry and entry velocity. For example, simulations involving steady, laminar flow of a perfect gas or a gas in thermochemical equilibrium over a blunt, spherically capped cone have the greatest confidence levels, with uncertainties predominantly associated with discretization errors. For such cases in the supersonic to hypersonic regime, aerodynamic coefficients would generally be expected to be well within two to three percent. The uncertainty errors associated with heating rate predictions in a high energy flow in thermal and chemical nonequilibrium for the same geometry will be much higher; but no blanket statement can be offered for all codes under all conditions.

\section{Margins - A Sample Case}

Figure 4 is a plot of heating margin estimates for the Stardust sample return capsule. These margin estimates are based on fully catalytic, non-ablating, axisymmetric Navier-Stokes calculations performed for the design of the capsule. For the forebody, the major sources of margin are from the computation (physical modeling and discretization errors) and angle-ofattack effects. A $25 \%$ computational margin is estimated for the forebody and $10 \%$ angle-of-attack effect is estimated for the shoulder. These margins are cumulative. Thus, at the shoulder, the adjusted heating value is 1.38 times larger than the zero margin value. In the afterbody region, the uncertainties are much higher. Unknowns related to turbulence, ablation, angle-of-attack, and the laminar wake heating computations are all major contributors to the design margins. At the PICA/SLA seal which is the joint between the forebody and afterbody TPS, the design margin is a factor of 4.5 .

To estimate and develop an acceptable risk assessment for an entry vehicle requires design margins. For example, a TPS designer wants to know the error 


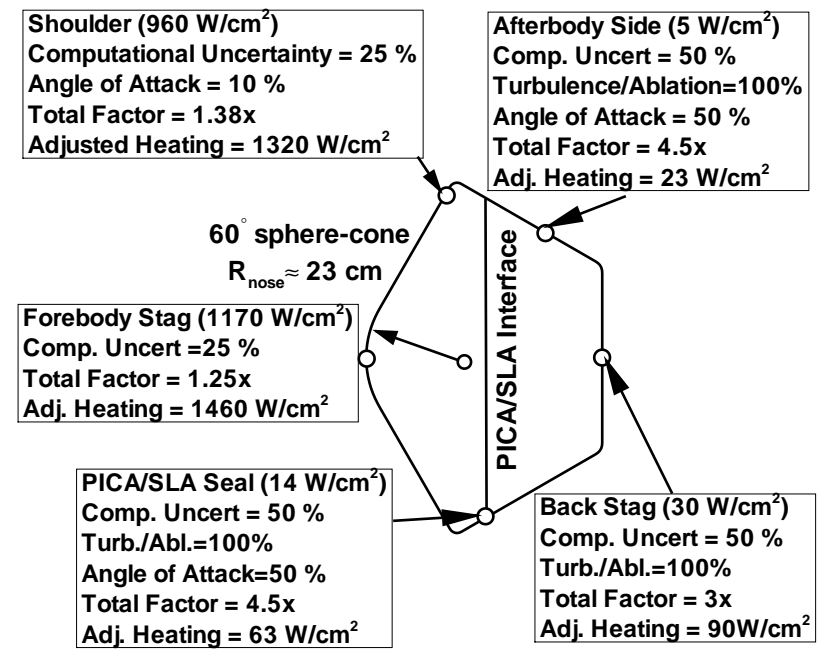

Fig. 4 Approximate entry heating design margins for the Stardust Sample Return Capsule

bars on an entry heating calculation to determine TPS mass margins. At present, a formal methodology does not exist for determining computational aerothermodynamic design margins. The current state-of-the-art consists of general estimates based on comparisons with flight data, experimental data, previous design experience, atmospheric uncertainties, and limited parameter studies. This "ad hoc" approach leading to the estimates in Fig. 4 is very limited.

A formal heating margin analyis methodology is possible using the approach employed in trajectory analysis. In trajectory analysis, a state vector, consisting of important simulation parameters, is selected. Next, uncertainties in the state parameters about a marginal value are estimated. Finally, perturbed combinations of the state vector parameters are selected via a Monte Carlo approach and a large number of trajectory analyses are performed. Variations about the reference trajectory determine design margins. For a Navier-Stokes heating calculation, parameters relating to the physical models such as chemical rates, transport coefficients, Schmidt number, and surface catalycity could be selected and their associated uncertainties could be estimated. Then, combinations of these parameters would be selected followed by a large number of Navier-Stokes calculations. For a simple geometry such as the forebody of a sphere and a limited chemistry set such as five species air, this type of analysis, while computationally intensive, is possible with present day computers.

\section{Codes}

The following codes are representative of capabilities available for hypersonic flow analyses, including options for thermochemical equilibrium and nonequilibrium. It is not an all inclusive list; rather, it is restricted to codes with which we have application experience.
LAURA (The Langley Aerothermodynamic Upwind Relaxation Algorithm)

Inviscid flux definition in LAURA employs Roe's averaging $^{29}$ and Yee's Symmetric Total Variation Diminishing (STVD) ${ }^{133}$ for second-order accuracy away from discontinuities. Harten's entropy fix (eigenvalue limiter) is applied across cell faces. Special variations of the limiter are employed across viscous dominated boundary and shear layers. These treatments overcome problems often encountered with the baseline Roe's method regarding the "carbuncle" phenomenon or the baseline Harten's method in which numerical dissipation (proportional to a large eigenvalue) competes with physical dissipation. Central differences are used to define viscous flux. Point-implicit relaxation of the steady form of the conservation laws at each control volume in a computational plane is implemented; sweeping from plane to plane in a block and from block to block across the entire domain of interest. Consequently, the relaxation algorithm is Jacobi within a plane, and Gauss-Seidel from plane to plane. The STVD formulation of the anti-dissipative, second-order corrections are uniquely well suited to point-implicit relaxation of the steady equations; other formulations have been found to be less robust in this context. Evaluation of the inviscid Jacobian with eigenvalue limiter parameter $\epsilon$ set to 0.5 increases robustness of the relaxation scheme without detriment to solution accuracy.

The LAURA code is unique among hypersonic flow solvers in two regards. First, it is the only known flow solver to exploit macrotasking on Cray computers. Macrotasking refers to parallel execution of code on a shared memory machine on a subroutine level. (In contrast, microtasking and autotasking generally occur on a do loop level within a single subroutine.) The use of macrotasking, along with provisions for asynchronous relaxation, enable exceptionally high average concurrency of tasks for a multitasked job. Asynchronous relaxation also enables physically motivated load balancing in which CPU cycles are concentrated in regions known to converge more slowly (e.g. separated flow regions preceding deflected body flaps, near wake). Second, a built in grid adaption routine simultaneously aligns the outer boundary of the computational grid with the captured bow shock and enforces near wall grid resolution required for aeroheating analyses. This feature greatly simplifies the grid generation process for multiple cases over a wide range of Mach numbers, Reynolds Numbers, and angles of attack on a single configuration.

The basic features of LAURA.4.1 (214 page user manual ${ }^{134}$ ) include options for five thermochemical kinetic models for 11 species air, two equilibrium air models, ${ }^{53,56}$ two-temperature thermal model (TNE2C), two algebraic turbulence models, six models for wall catalysis, a radiative equilibrium wall, discretiza- 
tion on up to six, simply connected, structured blocks, and mesh sequencing. Solutions of the Navier-Stokes, Thin-Layer Navier-Stokes, and Euler equations can be implemented. Solution Jacobians may be stored out-of-core for significant reduction (as much as factor 2 - function of number of unknowns) in memory requirement. New features offered in LAURA.4.4 (user manual not yet updated) include options for Martian atmospheric chemistry, 1000 structured blocks with integer stride connectivity (175 block solution tested), and post-processing files generated to support integralboundary-layer analyses of variations in surface catalysis and emissivity of the thermal protection system. A PVM/MPI version of the code is being tested which currently supports all of the options in LAURA.4.1 and most of the options in LAURA.4.4.

Multigrid relaxation had been tested on an earlier version of LAURA. ${ }^{44}$ In some cases, it yielded converged solutions in $1 / 3$ of the computational time at the expense of increased storage. However, this option is not currently supported because of memory overhead requirements.

Code validation runs for LAURA have been documented with STS flight data ${ }^{49,135}$ as well as with other flight test and ground based data. ${ }^{136,137}$

\section{GASP (The General Aerodynamic Simulation Program)}

Inviscid flux definition in GASP employs several options, including Roe's and Van Leer's upwind biased formulations and central differencing with artificial viscosity. Central differences are used to define viscous flux. A rich variety of options are supported for convergence of both steady and unsteady NavierStokes, Thin-Layer Navier-Stokes, Parabolized NavierStokes, and Euler equations. These options include mesh sequencing, preconditioning, approximate factorization (AF), Line Gauss Seidel (LGS), Generalized Minimal Residual (GMRES), ${ }^{138}$ mesh sequencing and multi-grid. Both algebraic and two-equation turbulence models with wall function options are supported. Generalized Zonal-Boundary Interpolation is supported across zonal intersections defined by a single logical boundary. Parallel processing is supported on shared memory computer architectures. A comprehensive set of thermochemical kinetic models is offered for air chemistry, hydrogen-air combustion, and hydrocarbons in a database containing 455 reactions and 34 species. Thermal nonequilibrium may be modeled using a separate vibrational temperature for each molecule (TNEM-V) or a lumped vibrational temperature common to all molecules (TNE2-Pv). The comprehensive GASP V3 Users Manual ${ }^{139}$ (654 pages) and the Graphical User Interface (GUI) for problem setup and data manipulation make GASP more user friendly.

The GASP code has been validated at hyper- sonic, nonequilibrium flow conditions with STS heating data. ${ }^{140}$

\section{GIANTS (Gauss-Seidel Implicit}

Aerothermodynamic Navier-Stokes code with Thermochemical Surface conditions)

The GIANTS $\operatorname{code}^{69}$ is a laminar, 2D/axisymmetric, full Navier-Stokes code which simulates thermal and chemical nonequilibrium. It was developed to accurately simulate the flowfield and surface boundary conditions characteristic of a pyrolizing or ablating TPS. Loosely coupled with a material response code, it has been used to perform aerothermodynamic analysis and TPS sizing for Mars and Earth entries; GIANTS was the primary TPS analysis code for the Mars/Pathfinder and Stardust heatshield designs. GIANTS has been coupled with a radiation module, NOVAR (Nonequilibrium Optimized VectorizeAble Radiation process model), and validated against data from the Fire II flight experiment. Also, code to code comparisons between GASP, LAURA, and GIANTS have been performed; for similar flow modeling (transport, chemistry, etc.), the codes agree well.

GIANTS employs a Gauss-Seidel line relaxation technique based on the work of Candler and MacCormack $^{141}$ and is a derivative of a code developed by Candler. It employs species density equations and finite rate chemistry to model chemical nonequilibrium, a vibrational energy equation to simulate thermal nonequilibrium, a bifurcation formulation for species diffusion, a modified Steger-Warming inviscid flux formulation and central differences for the viscous fluxes. The method is extremely efficient and robust for calculating hypersonic blunt-body flowflields. Efficiency and robustness are important because the integrated heatload is needed to size the TPS; therefore, heat transfer predictions are required at many points along an entry vehicle's trajectory.

\section{DPLUR (Data-Parallel Lower-Upper Relaxation)}

DPLUR $^{142}$ is based on a variation of the LU-SGS ${ }^{34}$ algorithm that is designed to optimize performance on a single-instruction, multiple-data (SIMD) computer architecture. The program seeks to exploit builtin, optimized interprocessor communications paths by appropriate assignment of data to processors and requiring only nearest neighbor communication. The method has been applied to inviscid, single block representations of the forebody of the X33 as well as other geometrically simpler but physically more complex simulations. ${ }^{60,132}$ Some aspects of the method may not translate well into unstructured grids because of the architectural constraints on nearest neighbor mapping. 


\section{FELISA_HYP}

The FELISA_HYP ${ }^{143}$ code employs an unstructured grid algorithm specifically constructed for robust, hypersonic flow simulation. It is a finite-volume based formulation that employs an efficient edge data structure. Second-order accuracy is maintained in smooth regions using linear reconstruction following MUSCL concepts. ${ }^{28,144}$ The Local Extremum Diminishing criteria $^{145}$ is used as a limiter near flow discontinuities. A simple, forward Euler explicit time stepping is used to relax the equations. The code is currently limited to inviscid flows. Options for equilibrium air chemistry are available.

In spite of its current limitation to inviscid flows, FELISA_HYP has proven particularly valuable in the CFD design environment because of the relatively quick grid generation capability. In the X-33 design Phase I, the FELISA grid tools could be applied by an experienced user to generate unstructured surface and volume grids in four days (first configuration took 1.5 weeks). In comparison, a multiple-block structured grid for LAURA took approximately four weeks to construct using state-of-the-art grid generation tools and expertise (first configuration took 6 weeks). (The LAURA grid required boundary-layer resolution for viscous flow, but this additional constraint is thought to add less than $30 \%$ penalty to the grid generation costs.) The actual FELISA_HYP solver is somewhat slower than the inviscid version of LAURA; however, the FELISA_HYP solver would finish several inviscid solutions on a new configuration before LAURA could even get started with a usable grid.

\section{OVERFLOW}

The OVERFLOW code is not specifically constructed for hypersonic flow simulations. The code is unique, however, in its use of overset structured grids to discretize complex configurations. This degree of freedom should decrease the grid generation time for entry vehicle design, provided that no special provisions are required for resolution of strong shocks traversing the overset grid regions. OVERFLOW has been used in high supersonic freestream conditions. ${ }^{146}$ The chemistry model does not now include equilibrium or nonequilibrium option, but does allow for frozen flow of multiple species (single phase), loosely coupled to the flow equations, to approximately account for solid rocket booster (SRB) plume effects on Shuttle ascent. The flow equations have a variable gamma (mixture of perfect gases/frozen chemistry) capability. The hypersonic simulation capability is not very mature at present.

\section{D Non-Body-Fitted Cartesian Euler}

The 3D Non-Body-Fitted Cartesian Euler flow solver $^{147}$ (see Gridding Strategies), like OVERFLOW, is not specifically constructed for hypersonic flow simu- lations. The flow relaxation algorithm uses a JamesonSchmitt-Turkel scheme ${ }^{148}$ using multi-stage RungeKutta integration and central differencing with added second- and fourth-order artificial viscosity. The scheme employs a feature detection algorithm which provides for cell enrichment across strong shocks. Although the code is currently restricted to perfect-gas simulation, it has been applied to a Mach 10 flow simulation over an X-33 configuration. Computed results for aerodynamics appeared to be of acceptable quality for preliminary design study; extensive validation and grid refinement tests are still required. It appears to be competitive with the FELISA_HYP unstructured approach; however, more comprehensive studies are required.

We believe it is worthwhile to bring codes like OVERFLOW and the Cartesian Euler to bear on hypersonic simulation problems so that the tradeoffs on various approaches to flow discretization (unstructured, patched structured, overset structured, Cartesian unstructured) can be understood and perhaps recognize synergistic, hybrid approaches that best meet design needs.

\section{Applications}

For simple geometries (e.g. capsules, planetary probes) CFD methods can generally define surface and volume grids and generate a matrix of solutions faster than experimental, ground-based methods. Support for $\mathrm{COMET}^{112}$ confirms this observation. As geometries grow in complexity, the situation reverses. At present, ground-based experimental methods can construct and test relatively complex configurations across a matrix of Mach numbers and Reynolds numbers much faster than structured, viscous CFD simulation methods. Unstructured, inviscid simulation matrices can be filled on a time frame that is only beginning to be competitive with ground-based methods. (Ultimately, if a fine-grained test matrix is required, involving sweeps in pitch, yaw, roll, and control surface deflections for a set configuration, even the unstructured, inviscid methods are not competitive with ground-based methods. Of course, the converse observations can be made for simulation matrices that extend beyond the Mach - Reynolds number capabilities of ground-based facilities.) Experience in Phase I and II for X-33 confirms these observations. Neither ground-based nor computational analyses have a lock on fidelity of simulation in the hypersonic flight environment.

The applications that follow describe the role computational aerothermodynamics has played in defining the aerothermodynamic environments for a variety of configurations and physical modeling requirements ranging from simple to complex. The examples predominantly reflect the authors' personal applications experience. 


\section{COMET/METEOR}

Aerodynamics and surface heating for the Commercial Experiment Transporter (COMET) at several points along its trajectory on return from Low Earth Orbit were calculated with LAURA and a Direct Simulation Monte Carlo method (DSMC). ${ }^{112}$ The COMET module (later renamed METEOR) has no active control system, and relies entirely on aerodynamic forces for stability and proper orientation during its maximum heating pulse. The aerodynamic data base was used within a six degree-of-freedom trajectory code to define a splashdown footprint. A Direct Simulation Monte Carlo method was used to define the flowfield in a transitional, rarefied regime (above $90 \mathrm{~km}$.) The LAURA program was used to define the flowfield in the transitional to continuum regime (below $90 \mathrm{~km}$.) Wake flows were included prior to the peak heating point because of the large initial angle of attack. Continuum and rarefied aerodynamic predictions for lift, drag, and moment were in good agreement at $90 \mathrm{~km}$. Thermochemical nonequilibrium models including 7 species for air were used down to Mach 15. Both viscous and inviscid solutions were used below Mach 15 . Wake flows were included at Mach 1.5 to account for important base flow effects on aerodynamics. A matrix of 46 solutions was completed between February 14 and March 23, 1995. This matrix included 10 reacting, viscous flows with wake; 13 reacting, viscous flows without wake, 6 perfect gas, viscous flows with wake, and 17 perfect gas, inviscid flows without wake. Angles of attack varied from 0 to 90 degrees. A solution adaptive grid was employed to swing the extended grid in the wake around the body behind the base at zero degrees to off the side at 90 degrees. Maximum job size was $71.2 \mathrm{MW}$ on the $\mathrm{C}-90$ and required 8.8 hours on a $72 \times 36 \times 64$ cell domain. Actual time on the computer for this case was only 0.98 hours because of extensive use of asynchronous macrotasking relaxation. The large average concurrent CPU usage enabled fast turnaround for this large matrix of cases.

Computed results were obtained prior to initiation of the wind tunnel test program and were in excellent agreement with wind tunnel data at Mach 6 (Fig. 5). Mach number independence for blunt body aerodynamics with minimal high temperature gas effects is evident in these results. Flight data is not available because the mission had to be aborted on ascent.

\section{Mars Pathfinder}

CFD analysis of the Mars Pathfinder was the predominant source for aerodynamic coefficients and heating environment. ${ }^{27,51}$ The spherically blunted, 70 degree half angle cone shape is very similar to the earlier Viking probe. While a significant data base from the Viking Project was used, the entry parameters for Pathfinder (velocity and trim angle-of-attack) ${ }^{149}$ differ significantly from Viking.

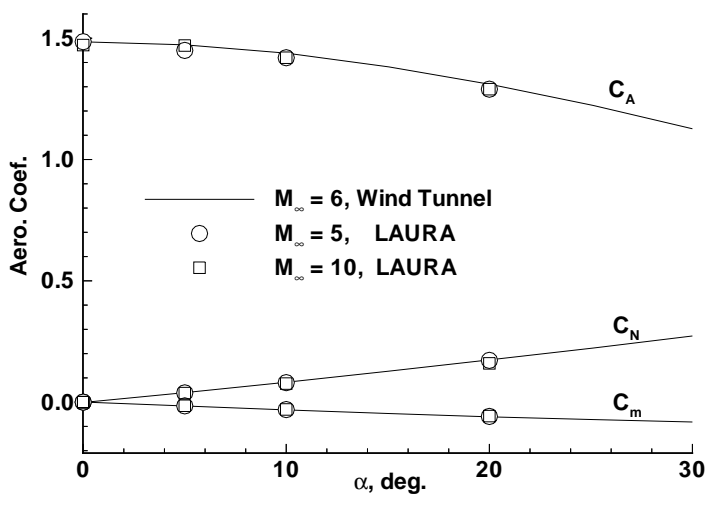

Fig. 5 Computed aerodynamic coefficients for the COMET re-entry vehicle at the Mach 5 and 10 trajectory points are compared to ground-based experimental data at Mach 6 .

This simple shape showed significant gas chemistry effects on aerodynamics. As the Mars Pathfinder probe descends through the Martian atmosphere the minimum value of the post-shock effective $\gamma$ first decreases from frozen gas chemistry values $(\approx 1.333-$ assumed effective 6 degrees of freedom in linear triatom) to equilibrium values (1.094) corresponding to a velocity of $4.86 \mathrm{~km} / \mathrm{s}$. As the probe continues to decelerate through an equilibrium post-shock gas chemistry regime, the value of $\gamma$ increases again, until reaching its perfect gas value of 1.333 at parachute deployment $(0.42 \mathrm{~km} / \mathrm{s})$. At small angles of attack $\left(\alpha<5^{\circ}\right)$ the sonic line location shifts from the shoulder to the nose cap and back again on the leeside symmetry plane because of the change in $\gamma$ and the cone half-angle of $70^{\circ}$. At $2^{\circ}$ angle of attack, the flat, leeside pressures approaching the shoulder (when the sonic line sits over the nose) can exceed the rounded windside pressures approaching the shoulder (when the sonic line sits over the shoulder). The net effect of this crossover distribution near the shoulder tends to pitch the probe to higher angles of attack. Conditions for a positive, destabilizing moment coefficient derivative $C_{m, \alpha}$ occur twice in the Mars Pathfinder mission (see Fig. 6) as determined by the viscous, thermochemical nonequilibrium flow simulations used in this study. The first occurrence $\left(7.5>V_{\infty}>6.5 \mathrm{~km} / \mathrm{s}, 51>h>37\right.$ $\mathrm{km}$, vicinity of peak heating for this trajectory) results from the transition in the sonic line location as a function of gas chemistry changing from nonequilibrium to equilibrium. The second occurrence $\left(4.0>V_{\infty}>3.1\right.$ $\mathrm{km} / \mathrm{s}, 25>h>22 \mathrm{~km}$ ) results from the transition in the sonic line location as a function of decreasing flow enthalpy in an equilibrium gas chemistry regime.

Sonic line movement also affects the heating distributions by altering the effective radius of curvature of the body. Peak heating tends to decrease as angle of attack increases for fixed freestream conditions 


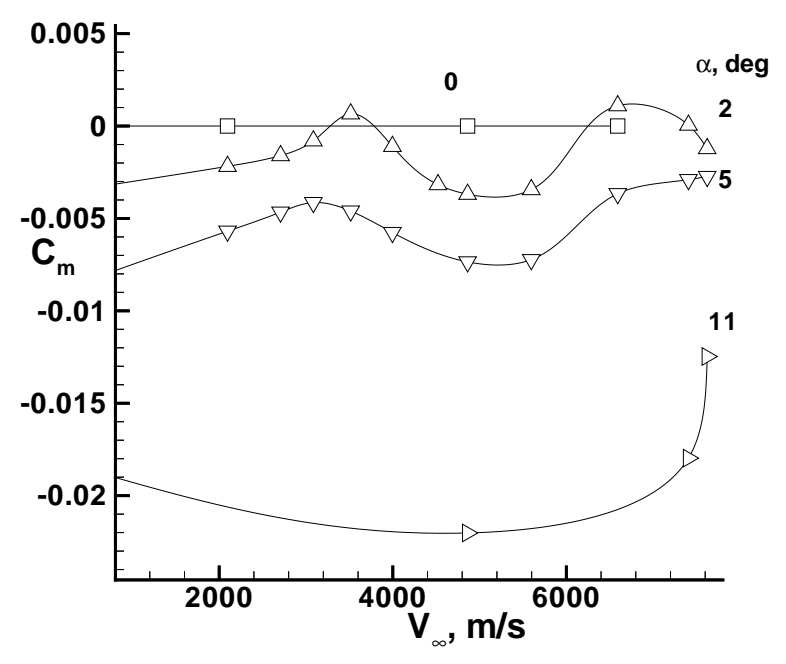

Fig. 6 Pitching moment coefficient versus velocity at several angles of attack $\alpha$ for the Pathfinder entry into the Martian atmosphere.

on the Mars Pathfinder probe because the body appears blunter to the oncoming flow. However, the drag coefficient decreases with increasing angle of attack, so that the ballistic coefficient increases and the peak heating point along the trajectory may be more severe.

\section{Stardust}

Stardust is a Discovery class mission that is scheduled for launch in early 1999 and return to Earth in early 2006. The prime scientific objective of the mission is to rendezvous with Comet Wild-2, gather samples of cometary particles and return them to Earth for further analysis. The captured cometary particles are stored in the Stardust sample return capsule. The sample return capsule enters the Earth's atmosphere at approximately 13.5 kilometers/sec and decelerates to .6 kilometers/sec in two minutes. At 3 $\mathrm{km}$, a parachute is deployed for a land based recovery in Utah. Significant milestones for the mission are the first attempt at a sample return beyond the Moon and the fastest Earth entry of a human-made object ever attempted. Further, a revolutionary new light-weight heatshield material, PICA, is being flight qualified on the Stardust mission.

The Stardust mission poses a number of aerothermodynamic challenges. Accurate predictions of the entry aerothermodynamic environment are needed for the following tasks: 1) forebody ${ }^{150}$ and afterbody TPS sizing, ${ }^{151} 2$ ) arc-jet testing for flight qualification of the heatshield, ${ }^{152,153} 3$ ) predicting the aerodynamic stability of the vehicle during entry, ${ }^{154,155}$ and 4) estimating the landing footprint for vehicle recovery. ${ }^{156}$ The flow environment for the high speed Stardust entry is complex; the sample return capsule heatshield is ablating and the flow is radiating. Further, shape change reduces the drag and must be considered for

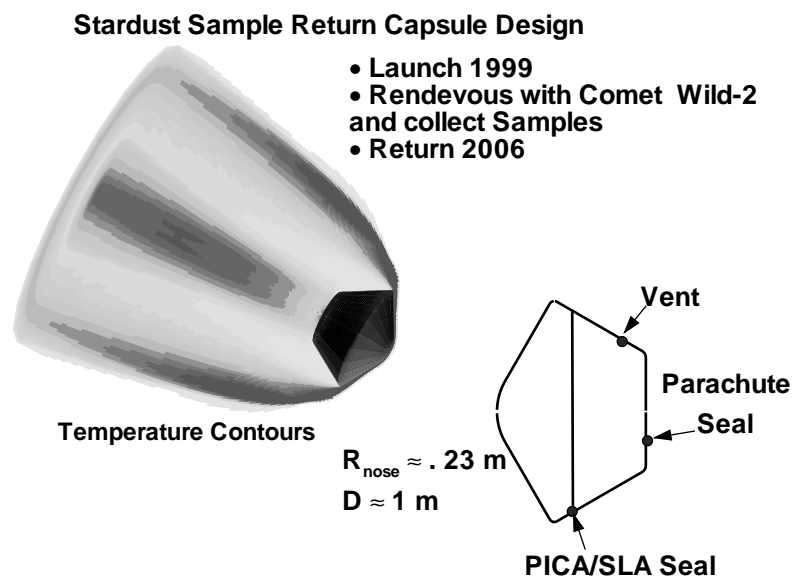

Fig. 7 Temperature contours at peak heating for the Stardust sample return capsule

the footprint calculations. For the Stardust sample return capsule design, these aerothermodynamic challenges are addressed using high-fidelity CFD analysis.

Aerothermodynamics estimates for the first two tasks, TPS sizing and arc-jet testing, are generated using the GIANTS flow solver, the FIAT ${ }^{157}$ material response code, and the NOVAR radiation code. These modules are loosely coupled to perform flowfield calculations with radiation and ablation for both the forebody and afterbody heatshields. Fig. 7 is a plot of temperature contours around the vehicle for a typical flow calculation. For the forebody PICA TPS sizing, an ablation and recession model is developed from arc-jet test results. The FIAT ablation model parameters are selected to reproduce recession and thermocouple data from a PICA arc-jet test series. For a given heat transfer rate, FIAT predicts surface temperature, surface mass fractions, and mass blowing rate; these values are used as GIANTS inputs. For the GIANTS flow simulation, ablation products from the forebody are allowed to propogate into the wake region; the afterbody contains a vent and contamination from the forebody ablation products is a concern. The afterbody heatshield is composed of SLA -561; mass injection from this material is not considered in the simulation.

Full-body flow calcuations are generated along the trajectory to define the heat pulse and size the TPS; the predicted thickness and total surface recession of the PICA heatshield are about $5 \mathrm{~cm}$ and $1 \mathrm{~cm}$ at the stagnation point. The effects of shape change are not considered in the flow simulation because the total recession at the stagnation point is only $4 \%$ of the nose radius. The drag reduction, however, from the shape change is considered in the footprint calculation.

For arc-jet testing and heatshield qualification, estimates of the non-ablative heat transfer are required; predicted values at a few vehicle locations are shown in Figs. 4 and 7. The peak non-ablative heat transfer at the nose assuming a fully catalytic surface is about 
$1300 \mathrm{~W} / \mathrm{cm}^{2}$ with radiation. CFD predicted surface heat transfer, shear, and pressure values are used to set arc-jet test conditions. Using these conditions, arc-jet models are tested to estimate the performance of various components of the heatshield design such as the seal between the forebody and afterbody heatshields, the parachute seal, the afterbody vent, and the forebody TPS at stagnation conditions.

Aerodynamic stability and the footprint calculation (items 3 and 4) are critical to the success of the Stardust mission. These issues are addressed using a 6-Degree of freedom trajectory analysis code (POST). The accuracy of the POST simulation and dispersion analysis is a direct function of the fidelity of the aerodynamic database from initial atmospheric entry through parachute deployment. The flow regimes spanned by the aerodynamic database are free-molecular, transition, hypersonic, supersonic, transonic, and subsonic. Thus, a wide variety of simulation tools and resources are employed. Direct Simulation Monte Carlo (DSMC) is used in the freemolecular and transition regimes. High-fidelity CFD (LAURA) and low-fidelity Newtonian codes are used in hypersonic flow regimes. Finally, both CFD and wind tunnel results are employed in the supersonic, transonic, and subsonic flow regimes.

The packaging of the Stardust sample return capsule in combination with the light-weight forebody PICA heatshield produced a number of c.g. related stability issues. These issues were identified with the combined trajectory and aerodynamic analyses. For example, in the free-molecular and transitional flow regimes, a potential for the vehicle to flip around was identified. Another issue addressed was the Mach number for parachute deployment which is a strong function of the transonic and subsonic dynamic stability of the sample return capsule.

\section{Galileo}

The Galileo probe was designed to withstand the harshest environment ever encountered by any planetary probe. ${ }^{158}$ One of the major objectives was to determine the atmospheric structure of Jupiter. ${ }^{159}$ The probe entered the Jovian atmosphere at a relative velocity of $47.5 \mathrm{~km} / \mathrm{s}$. and within the first $100 \mathrm{sec}$. after entry, the probe decelerated to less than $1 \mathrm{~km} / \mathrm{sec}$. The $45 \mathrm{deg}$. blunted-cone probe was designed to withstand stagnation point peak-heating rate of $30 \mathrm{~kW} / \mathrm{cm}^{2}$ and total heat-load of $300 \mathrm{~kJ} / \mathrm{cm}^{2} .107$ The forebody heating was mainly due to shock-layer radiation during reentry. These environments were originally defined by state-of-the-art computational aerothermodynamic methods of the 1970's and early 1980's as represented by several papers ${ }^{160,161}$ and reviewed by Howe et. al. ${ }^{162}$

The determination of the structure of the Jovian atmosphere from the flight measurements requires ac- curate prediction of the the aerodynamic forces including drag coefficient of the probe during entry. ${ }^{159}$ Ballistic range measurement coupled with CFD analysis ${ }^{163}$ established the drag coefficient in the postand pre-ablative regions. At present the drag coefficient estimation is limited to heurestic modeling during the ablation phase. Though the recession measurement during flight helps in determining the mass loss and shape change, we have yet to establish valid methodologies in determining the aerodynamic forces and moments during the ablative phase. Since accurate prediction of the aerodynamic forces and moments are not only necessary for probe design and trajectory determination, these predictions form the basis of "Science Experiments" and it is a challenging task when the environment is as harsh as the one encountered by the Galileo probe.

A harder problem during the probe design was the heat-shield thickness determination ${ }^{158}$ and this poses a formidable challenge even today. The ablative heatshield material selected was carbon-phenolic and a number of modeling studies were used to determine the expected recession of the forebody heat-shield. The ablative response modeling of the heat-shield involved a coupled thermal environment prediction, including radiation and turbulence models with in-depth material response. During the entry, the heat-shield experiment allowed the determination of the actual surface recessions. The flight measurements compared with deign predictions showed the stagnation point recession to be significantly less, whereas the frustum recession far exceeded the predictions. The challenge facing our community today is to build and verify hifidelity analysis tools that can accurately predict the recession rates for future missions to Jupiter, Europa and Neptune.

\section{STS}

CFD has worked primarily in a post flight, reactive mode regarding impact on the Shuttle operations and flight analysis. Several important studies are cited here because they are representative of the role CFD plays in this environment.

An apparent anomaly in the body flap effectiveness (relative to pre-flight data book) at high Mach number observed in STS-1 has been traced to small changes in pressure associated with changes in the specific heat integrated over a large, expansion area on the windside. ${ }^{135,164}$ Thermocouples on early Shuttle Orbiter flights have been used as a critical part of the code validation process, ${ }^{49}$ including effects of finite wall catalysis. The overset grid capability in OVERFLOW has been applied to a transonic, ascent flight condition for the complete Shuttle ascent vehicle, including an approximate treatment of real gas effects in the plume. ${ }^{165}$ A preliminary demonstration of the overset methodology for a supersonic flow of a perfect 


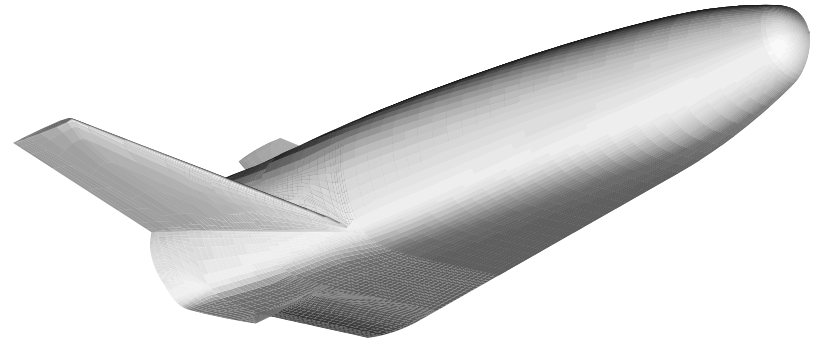

a) Windside

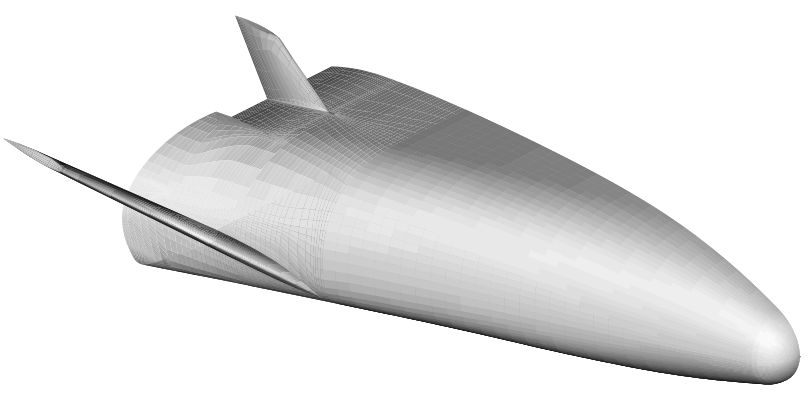

b) Leeside

Fig. 8 B1001 configuration (RLV)

gas involved a proposed canard at Mach 5.8 on the Shuttle. ${ }^{146}$

\section{X-33 and Reusable Launch Vehicle - Phase I}

Numerical simulations of hypersonic flow over preliminary configurations proposed by Lockheed Martin, McDonnell Douglas, and Rockwell for a Reusable Launch Vehicle (RLV) and X-33, a technology demonstrator for the RLV, were conducted. The process for defining the aerothermal characteristics of all three configurations by independent CFD teams was essentially the same. The process, as applied to the Lockheed Martin configuration, is described below.

The simulations were executed using both chemical equilibrium and nonequilibrium gas models. Simulations were generated over six representative trajectory points for descent of the B1001 RLV configuration in order to establish traceability of aerothermodynamic design issues. Simulations were generated over five representative trajectory points for descent of the B1001A X-33 configuration. Trajectory points for simulation were chosen near peak heating and peak dynamic pressure; other points were selected on the basis of convenient anchors for Mach number and angle of

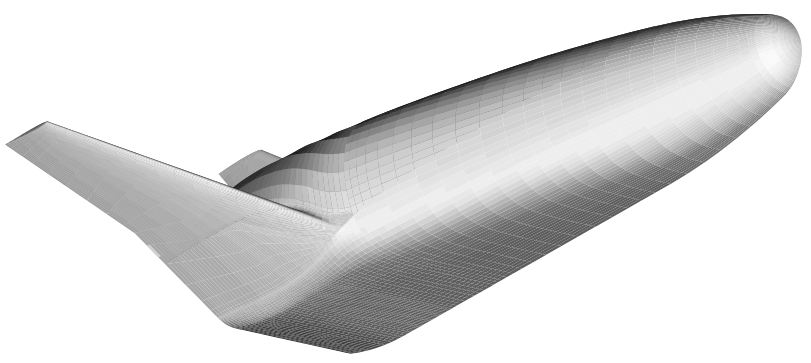

a) Windside

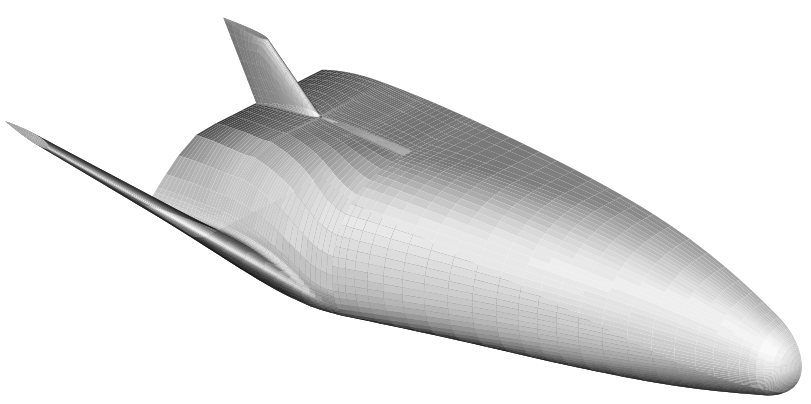

b) Leeside

Fig. 9 B1001A configuration (X-33)

attack variation. Representative surface heating, temperature and pressure distributions were provided to the design team, some examples of which are presented here. Procedures for incorporating CFD solutions into engineering code (MINIVER) format for subsequent use by the thermal design team are also discussed.

\section{Configurations and Grid}

Configuration with designation B1001, presented in Fig. 8, was used in the RLV simulations. It has a reference length of 1419.25 inches. Configuration with designation B1001A, presented in Fig. 9, was used in the X-33 simulations. It has a reference length of 752.2 inches which is 0.53 of full scale RLV. Both configurations assume a moment center at 0.66 reference length behind the nose. The vehicle geometries are identical to scale from the nose to upstream of the wing (hypervator) root. $\mathrm{B} 1001 \mathrm{~A}$ is tapered more toward the base to reduce base drag as compared to its predecessor. It has body flaps on wind and lee sides that terminate at the cowl trailing edge but extend across most of the base lateral dimension. B1001 has no control surface preceeding the central base region surrounding the aerospike engines. Instead, there is an expansion 


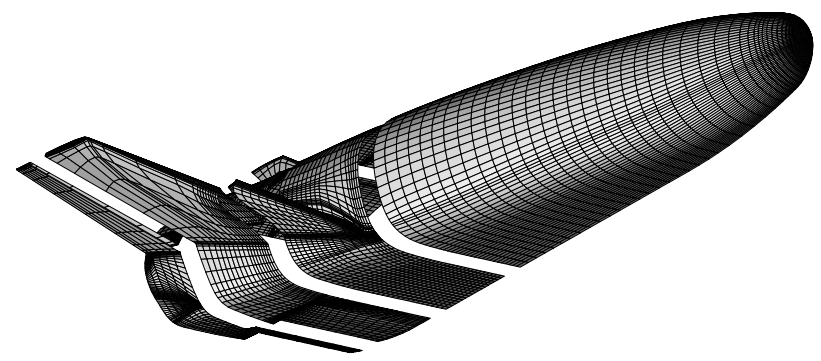

a) Windside

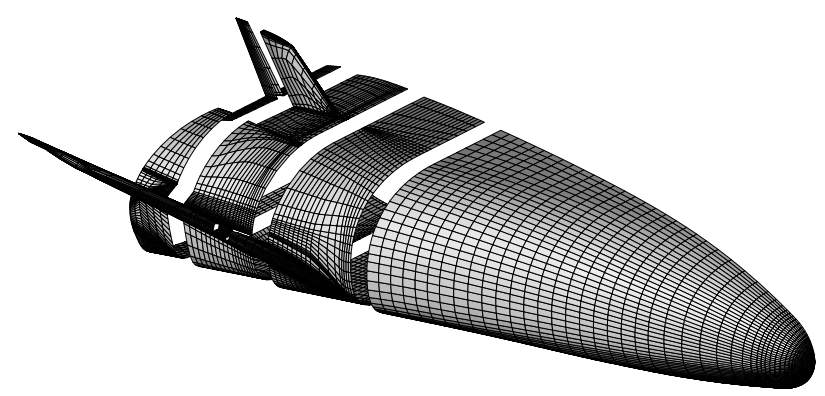

b) Leeside

Fig. 10 B1001 surface grid (RLV)

surface in the central region with outboard body flaps that extend past the cowl trailing edge. Parts of the body flap that extend past the trailing edge of the RLV (B1001) were not modeled in Phase I studies because body shape had already evolved to the B1001A based on wind tunnel test results. A set of simulations at a single body flap deflection (50 deg. - 30 deg relative to the waterline of the B1001A configuration) will be discussed.

Surface grids for B1001 (RLV) were constructed in four sections as shown in Fig. 10. Solutions were generated in each section sequentially in a block marching mode for B1001. The first section extends from the nose to the first terminal plane approximately 10 inches upstream of the wing (hypervator) root. The $\xi$ coordinate lines extend from the axis of the nose straight back to the terminal plane with roughly equal arc length between lines. The $\eta$ coordinate lines extend circumferentially around the body. The grid density in the first section is $(52 \times 64)$ cells.

The second section continues from the terminal plane of the first section to a second terminal plane that precedes the vertical tail on the leeside and juncture of the outboard body flaps and the inboard ex-

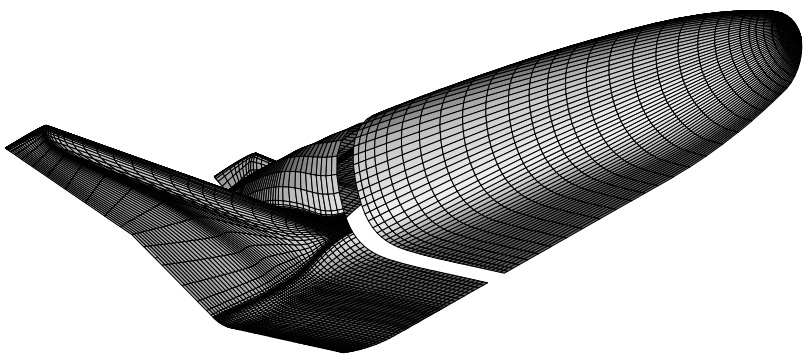

a) Windside

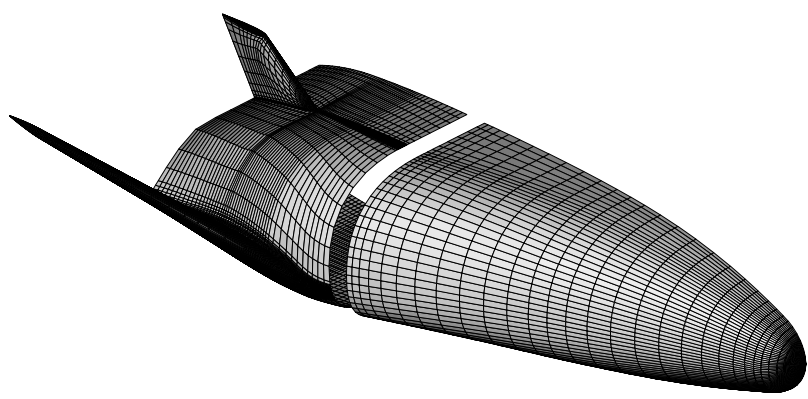

b) Leeside

Fig. 11 B1001A surface grid (X-33)

pansion surface. Additional circumferential $(\eta)$ coordinate lines are added in this section to define the wing (hypervator) and provide smooth transition of circumferential arc length in the vicinity of the wing / body junction. Some additional coordinate lines were also added to provide a smoother transition to the tail and bodyflap junction. The section was contructed with 9 blocks in the circumferential direction and a total density of 18 x 116 cells.

The third section extends from the second terminal plane to a third terminal plane at the bodyflap hinge line. Additional circumferential density was added to define the vertical tail and the outboard bodyflap /inboard expansion surface juncture. The section was contructed with 12 blocks in the circumferential direction and a total density of $18 \times 186$ cells.

The final section extends from the third terminal plane to the cowl trailing edge. The section was contructed with 23 blocks in the circumferential direction and a total density of $6 \times 293$ cells.

Surface grids for B1001A (X-33) were constructed in two sections as shown in Fig. 11. Lessons learned in the first gridding procedure and a simpler aft geometry enabled the simpler grid system. Solutions were gen- 


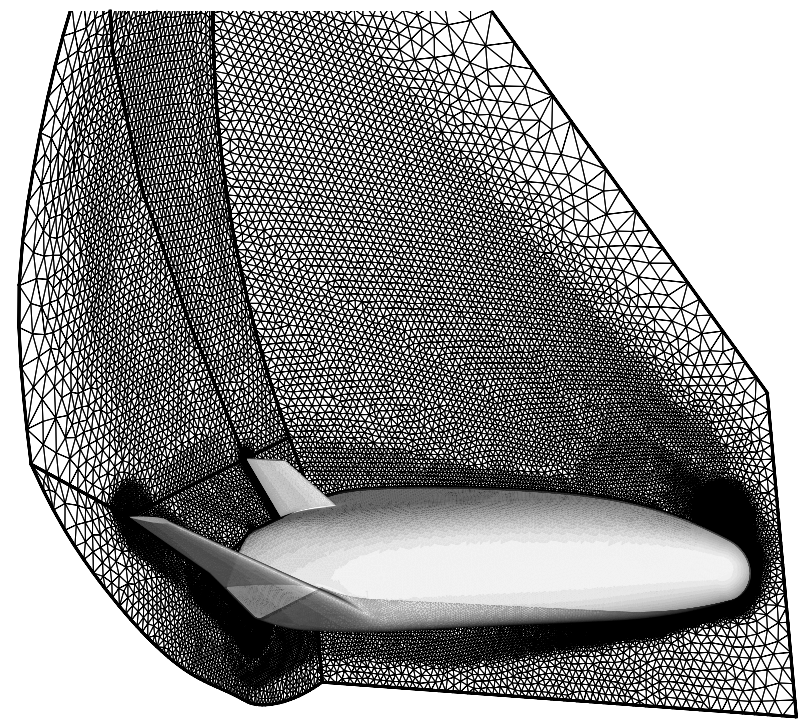

Fig. 12 B1001A unstructured surface and volume grid (X-33) from FELISA

erated in each section sequentially in a block marching mode for B1001A. In some cases, solutions were then regenerated in a fully coupled mode.

The first section extends from the nose to the first terminal plane approximately 10 inches upstream of the wing (hypervator) root. The $\xi$ coordinate lines extend from the axis of the nose straight back to the terminal plane with roughly equal arc length between lines. The $\eta$ coordinate lines extend circumferentially around the body. The grid density in the first section is $(64 \times 64)$ cells.

The second section continues from the terminal plane of the first section to the cowl trailing edge. Additional circumferential $(\eta)$ coordinate lines are added in this section to define the wing (hypervator) and provide smooth transition of circumferential arc length in the vicinity of the wing / body junction. Some additional coordinate lines were also added to provide a smoother transition to the tail and bodyflap junction. The section was contructed with 12 blocks in the circumferential direction and a total density of $100 \times 247$ cells.

In contrast to the structured grid used by LAURA, an unstructured grid used in FELISA analyses is shown in Fig. 12. This grid is for an inviscid application, but still reveals some of the requirements for relatively fine grid over the nose and in front of wing leading edges where the bow shock lies close to the body. FELISA has no automatic mechanism for collapsing the outer boundary to just in front of the bow shock as in LAURA.

\section{Thermal Analyses}

The most relevant validation data set(s) for X-33 heating rates include: (1) thermophosphor tests on the identical configuration at Mach 10 and Reynolds number 500,000 and total temperature 1000K; and (2)
STS-2 thermocouple data in flight between Mach numbers of 24.3 and 12.86, between Reynolds numbers of 171,000 to $4,387,000$, and between altitudes of 72.4 and $54.8 \mathrm{~km}$. These tests showed agreement with experimental data for laminar heating rate over most of the windside within $10 \%$ with some discrepancies as large as $20 \%$.

The computational aerothermodynamic analyses were focused on defining global temperature distributions around the RLV and X-33. Thermal analysis of the tanks required time dependent data in a readily accessible format as commonly provided by the MINIVER code. ${ }^{166}$ The required temporal resolution was much finer than the matrix of points considered by LAURA alone. The MINIVER code is capable of making reasonably accurate estimates of centerline heating distributions on vehicles like RLV and $\mathrm{X}-33$. However, three-dimensional flow effects ocurring off-centerline generally are not well-approximated by MINIVER analyses without some externally derived corrections. The necessary corrections are provided by LAURA at off centerline locations at the times defined in the CFD matrix.

Implementation of this procedure in phase I for both RLV and X-33 analyses occurred as follows. Heating and temperature distributions over the vehicle were generated by LAURA and compared with the windward centerline results from MINIVER. These comparisons established MINIVER as a reasonably accurate tool for the geometries and trajectories considered here in Phase I studies. Off-centerline values are keyed to centerline values of laminar heating rate in a relatively dense matrix of computational planes through plots of $q_{\text {Laminar }} / q_{\text {Laminar, } C L}$ as a function of spanwise location in the plane. This data is input to MINIVER in tabular form.

Transition to turbulence is assumed to occur for values of $R e_{\theta} / M_{e}$ between 250 and 300. A transition front is defined in LAURA across a computational plane. Turbulent heating levels are computed downstream of this plane and values of $q_{\text {Turbulent }} / q_{\text {Laminar }}$ are defined using earlier laminar solutions. These turbulent to laminar factors are also input into MINIVER in tabular form. Heating at any point on the body is then predicted by MINIVER by computing the windside centerline value at the same axial location, multiplying by an appropriately interpolated value for $q / q_{C L}$ for the spatial location on the body and temporal location along the trajectory, and applying an additional correction factor for turbulent flow if the transition criteria is exceeded.

Prediction of the transition criteria by LAURA and MINIVER along the windward centerline were in significant disagreement. LAURA predicts the threshhold transition criteria to occur earlier in the trajectory than MINIVER. Because these transition criteria have historically been derived from engineering code 


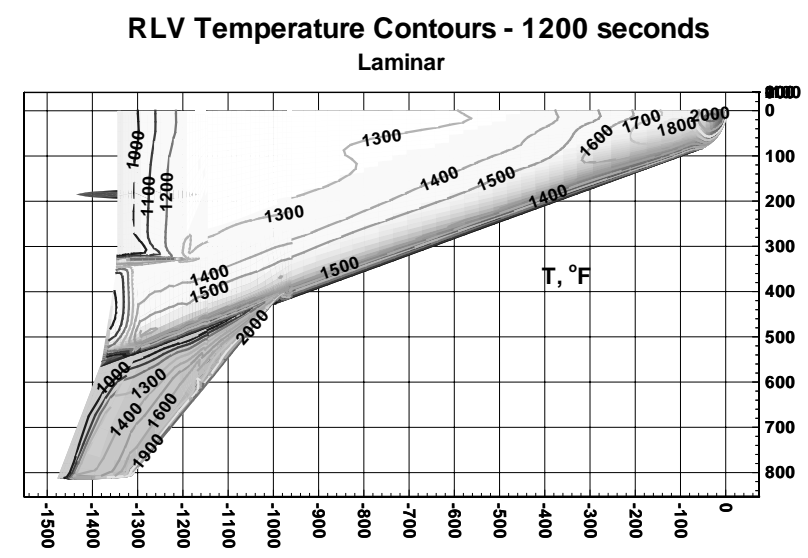

a) windside

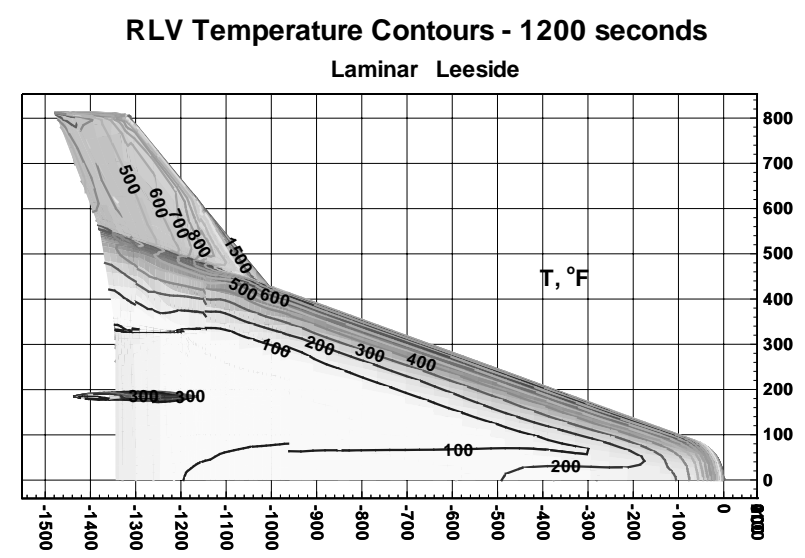

b) leeside

\section{RLV Temperature Contours - 1200 seconds Laminar}

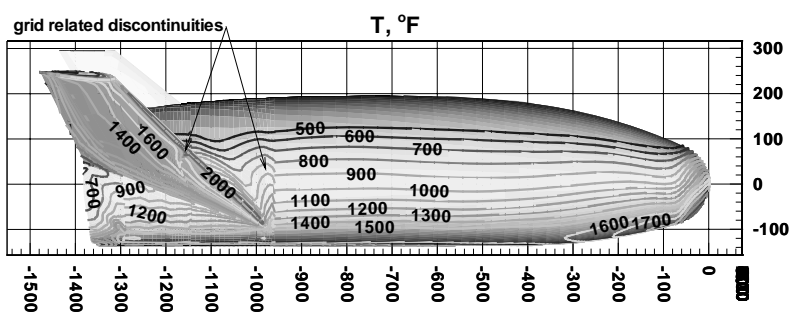

c) side

Fig. 13 Temperature contours on RLV for fully catalytic wall, laminar flow, at $1200 \mathrm{~s}$.

analyses like MINIVER, Phase I studies proceeded using MINIVER criteria. Establishment of a proper criteria is a subject of ongoing research. (See related discussion in Physical Models.)

Temperature maps of the vehicle as predicted by LAURA for the $1200 \mathrm{~s}$ point of the RLV entry are presented in Fig. 13. (All temperatures in these and

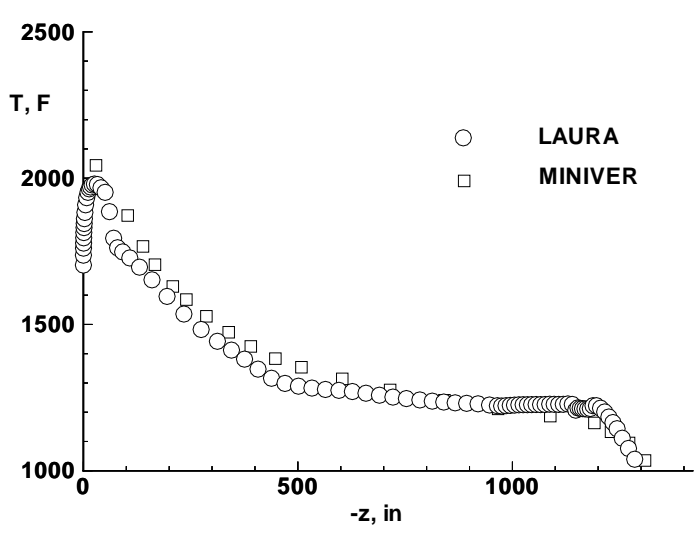

Fig. 14 Windside centerline comparisons of LAURA and MINIVER temperatures at the 1200 s trajectory point for the RLV.

subsequent figures are in degrees Fahrenheit.) Highest heating rates occur near the wing root but are only slightly higher than the stagnation point heating on the nose.

Solutions at this trajectory point are generated sequentially across four sections of the vehicle. Each successive section has more circumferential resolution to define the wing, tail, and edges of control surfaces. The solution was not run in a fully coupled mode; consequently, some cosmetic effects of domain interfaces are evident in the solution. Reasonably good agreement with the engineering code MINIVER along the windside centerline is demonstrated in Fig. 14.

\section{Body Flap}

The baseline configuration for B1001A has an expansion surface of approximately $20 \mathrm{deg}$. that leads to the cowl trailing edge above the engines and includes a stowed body flap. A flap deflection of 50 deg. (30 deg. into the unexpanded flow) was simulated to study effects on aerodynamics and heating using component isolation of the deflected flap and surrounding area. The initial solution came from an undeflected flap case. Enhanced grid around the deflected flap was implemented by partitioning the aft section into two streamwise sections with 14 blocks each. The section immediately preceeding the deflected flap was resolved into 28 streamwise cells by 149 circumferential cells distributed across 14 circumferential blocks. The section over the deflected flap was resolved into 22 streamwise cells by 173 circumferential cells distributed across 14 circumferential blocks. Approximately 30 hours of computer time were devoted to obtaining this solution, but the error norm never settled down during the simulation. Some initial difficulties were attributed to a transient reverse flow that set up over the edge of the flap in the exit plane. Vacuum boundary conditions ${ }^{49}$ were applied to survive this transient; nevertheless the solution never 


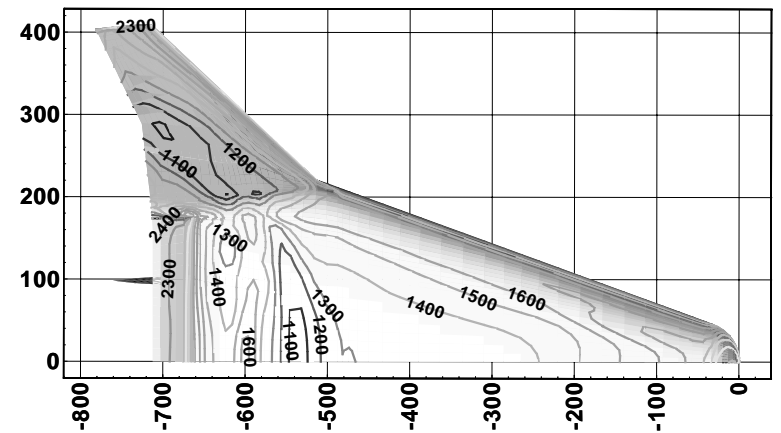

a) windside, fine grid

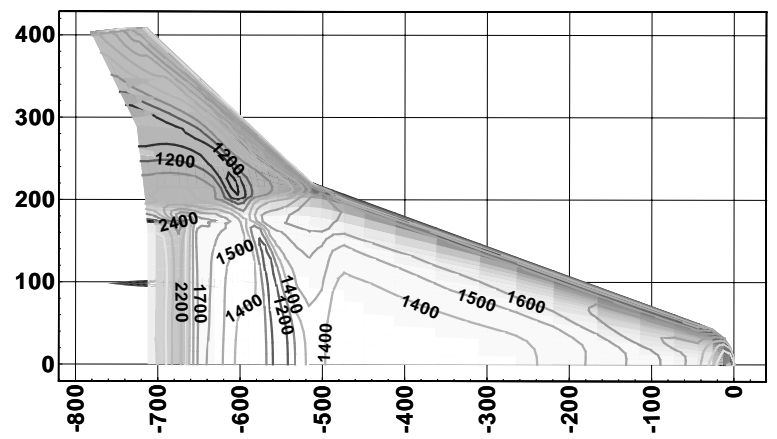

b) windside, coarse grid

Fig. 15 Temperature contours on X-33 for fully catalytic wall, laminar flow, at $370 \mathrm{~s}$ with a $50 \mathrm{deg}$. bodyflap deflection.

fully converged.

Previous experience in computing separated flow in front of ramps has indicated that such flows take a long time to set up. It appears that the separation bubble grows slowly with the upstream separation point moving further upstream as more mass is entrained. Coarsening the grid had been observed to speed up the solution process by providing a better initial condition for a subsequent fine grid solution. In the case of X-33, the coarse grid solution (every other point deleted) converged very quickly; however, the subsequent fine grid solution (all points restored) would rapidly evolve into an apparent unsteady flow within the bubble. (The LAURA simulation uses pseudo time advancement with large, constant Courant number and asynchronous relaxation; consequently, the evolution of the flow in the bubble is not simulated in a time accurate manner.) Embedded vortices appear within the bubble but change size and location in the simulation. Local hot and cold spots occur in the unsteady, fine grid solution (Fig. 15 (a)) that are not evident in the steady, coarse grid solution (Fig. 15 (b)).
The bodyflap was somewhat hotter in the fine grid solution than in the coarse grid though the outboard edge of the flap showed equivalent, high radiative equilibrium wall temperatures (2400 F). Application of a Baldwin-Lomax algebraic turbulence model across the separated flow region and the deflected flap also suppressed unsteady phenomena on the finest grid.

Simulation of unsteady separation in a hypersonic environment on a relatively complex configuration remains a challenge to state-of-the-art application tools. The results (steady vs. unsteady) are sensitive to numerical (grid-related) and physical (turbulent viscosity) dissipation levels. Turbulent viscosity in the present test is based on an algebraic model which is not appropriate for massively separated flow. Time accurate simulations involving two-equation turbulence models are planned for this case.

\section{X-33 - Phase II}

In the X-33 Phase I design, the aerothermal environment predictions were made for a preliminary trajectory (trajectory based TPS design) and configuration. In Phase II, trajectories and configurations have evolved to a final design at a pace faster than can be accommodated by CFD. Consequently, CFD simulations are performed at a number of discrete design points described by the variation in Mach number, angle-of-attack, and Reynolds numbers. These design points are selected to adequately span the design space for all possible trajectories. The CFD solution set for a given OML (Outer Mold Line) can then be used to determine the aerothermal heating and heatload for any trajectory within the design space quickly through interpolation with engineering codes. The aerothermal environment can be defined in a matter of minutes through this approach compared to weeks by the previous approach. Once the TPS subsystem is designed to a specific trajectory, the TPS margins can then be determined quickly by simulating the environment for other "off-nominal" trajectories to provide the desired margin. (A series of papers to be presented at the Aerospace Sciences Meeting in January 1998 detail the "Design-space CFD approach to TPS design" and other issues relevant to margins and errors.)

\section{Forebody to Cowl Trailing Edge}

Both LAURA and GASP codes have been used in predicting the aerothermal environment which will be used to design the TPS in Phase II. LAURA solutions are primarily used to define the forebody, body flap and the base region surface temperature at specific trajectory points on descent using a high fidelity grid. GASP solutions are generated for the forebody on a coarser surface grid but on a richer solution point matrix, based on complete trajectories and at critical design points. In general, for the grids used by the two solvers, the GASP and LAURA solutions are in good agreement over the majority of acreage on the 
X33. In fact, the predictions from the two solvers have been compared and the difference between the two predictions differ no more than $15 \mathrm{~F}$ under identical freestream conditions using the same grid in the nose region. The level of agreement between the two solvers is indeed gratifying considering that the numerical schemes and the procedures are different. Application of two codes by two groups (LAURA at Langley, GASP at Ames) at a few overlapping points provides an independent, critical check of predictions which enhance confidence in all of the CFD predictions. A brief review of the GASP and engineering code $\mathrm{HAVOC}^{167}$ application to X33 TPS design follows. More complete details will be presented in a series of papers to be presented in the AIAA Aerospace Sciences Meeting in January 1998.

The use of GASP solver towards the X33 TPS design is to generate high-quality, cost-effective solutions quickly. A series of grid refinement and grid sequencing studies were performed to determine the optimal grid points and convergence criteria for a prescribed tolerance for surface temperature, which is $25 \mathrm{~F}$ or less. The total number of grid points required to accomplish this was less than $(113 * 113 * 65=830,000)$ and a single converged reacting-air solution required 35 hr. on CRAY C-90. A maximum of 10 solutions per trajectory were generated to describe the aerothermal environment. The integration of the engineering code HAVOC coupled with the CFD determined the total heat-load and the surface temperature as a function of time.

The aerothermal environment can be defined independent of the trajectory and this approach is currently adapted in the X33 program. The design space can be discretized in an intelligent manner and a set of CFD solutions (approximately 40) can provide the aerothermal environment basis set. From these solutions and using engineering codes such as HAVOC, a complete trajectory based solution can be obtained in a matter of minutes. Such an approach can be integrated with trajectory optimization codes to determine the optimal trajectory from TPS material perspective.

\section{Body Flap and Wake}

Wake flow simulations have been implemented for X-33 (Phase II) in order to better assess bodyflap effectiveness and aerothermal loads on the aerospike engine on descent. In the present configuration (B1001F), the bodyflap extends past the cowl trailing edge into the wake. An accurate assessment of bodyflap effectiveness must allow for the flow to spill off the sides of the flap into the wake. The grids used in Phase II on the B1001F are very similar to those generated for the B1001A of Phase I.

Results are presented for a laminar, steady, nonequilibrium flow ( 5 species) on $\mathrm{a} \approx 4$ million cell grid

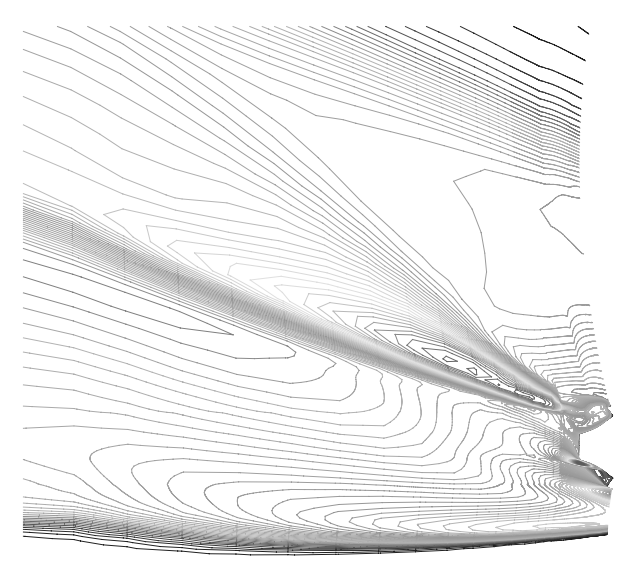

Fig. 16 Temperature contours in symmetry plane of X-33 wake at Mach 10.5 and $\alpha=26$ deg.

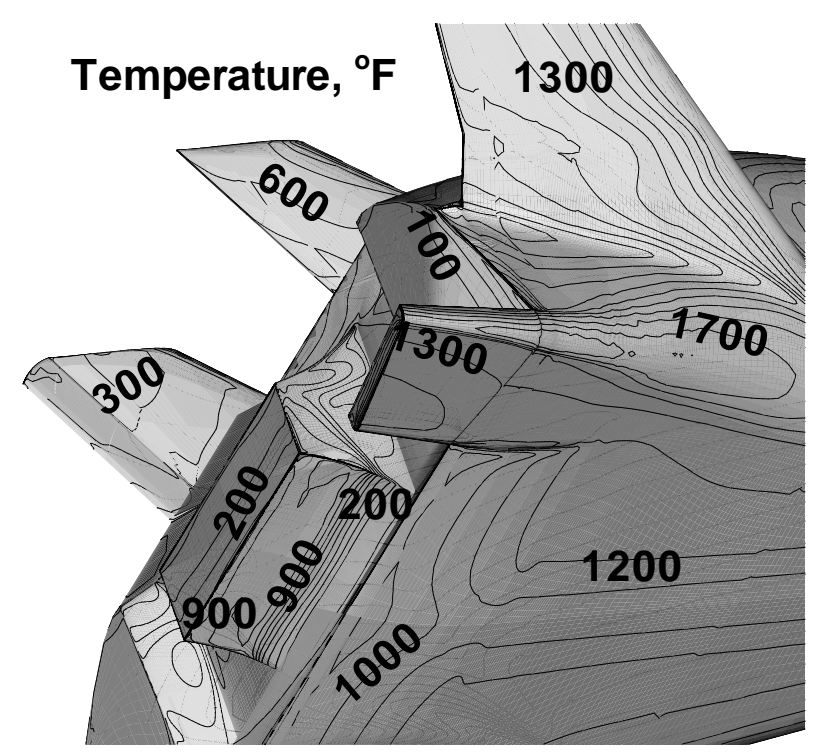

Fig. 17 Temperature contours in base region of X-33 wake at Mach 10.5 and $\alpha=26$ deg.

distributed across 175 computational blocks. The LAURA program requires $228 \mathrm{MW}$ of memory (180 MW if the Jacobians are stored on disc) and over 100 C-90 hours for a complete wake flow simulation. Extensive use of mesh sequencing and component isolation procedures were employed in generating these solutions. Surface heating levels changed less than $5 \%$ after the last grid doubling, with most of that change concentrated near component edges.

Figs. 16 and 17 show temperature contours in the plane of symmetry and base of the $\mathrm{X}-33$ wake region at Mach 10.5 and $\alpha=26 \mathrm{deg}$. The recompression shocks preceeding the wake core are evident in the figure. Impingement of the shear layer on the aft end of the aerospike is also evident. Surface temperatures are evaluated using radiative equilibrium wall bound- 
ary conditions. The implementation of this boundary condition assumed radiation into free space with no accommodation given for direct radiation from adjoining surfaces. ${ }^{168}$ The engine block temperatures are computed with emissivity of 0.12 , as compared to 0.8 for the bodyflap and 0.6 for all other surfaces. Consequently, the windside engine surfaces respond to a given heating rate with higher radiative equilibrium wall temperatures than other surfaces.

Fig. 18 shows the aerodynamic coefficients as a function of flap deflection angle measured in the Mach 20 Helium tunnel at Langley and computed at flight trajectory points. For zero flap deflection, there is little evidence of real gas aerodynamic effects as judged by the good comparison between computation at flight conditions and ground-based experiment. This trend was also observed in Phase I ground based experiments when comparing aerodynamics measured in Helium, air, and $\mathrm{CF}_{4}$. The configuration has a relatively flat windside surface as compared to the Shuttle Orbiter and shows considerably less sensitivity to real gas effects on aerodynamics with undeflected control surfaces. Bodyflap effectiveness as computed for flight conditions is stronger than indicated in the experimental data. This discrepancy is believed to a be caused by differences in the separation zone preceeding the deflected flap shock strength associated with the boundary-layer thickness approaching the flap and ratio of specific heats. Other relevant validation data sets for X-33 aerodynamics are: (1) STS-2 aerodynamic data measured in flight between Mach numbers of 24.3 and 12.86 , between Reynolds numbers of 171,000 to $4,387,000$, and between altitudes of 72.4 and $54.8 \mathrm{~km}$. and (2) STS-1 measurement of bodyflap deflection required for trim during a "pitching-moment anomaly". Computed aerodynamic coefficients were within experimental uncertainty for the STS-2 data points. The computed bodyflap trim angle for STS-1 was within $10 \%$ (1.5 deg.) of the recorded value at a $M_{\infty}=23$.

Computations that include the wake show flow recirculating back onto the leeside of the vehicle. As with the case of separated flow on COMET, ${ }^{112}$ extrapolation outflow boundary conditions on the leeside of a vehicle can falsely suggest that the flow stays attached to the trailing edge of the vehicle. Misprediction of this separation has little impact on hypersonic aerodynamics because of the very low relative pressure on the leeside at moderate to high angles of attack. For low supersonic to transonic flows, misrepresentation of leeside separation can have serious consequences on prediction of normal forces and pitching moment.

There is almost no validation data available for the simulation of aerodynamic heating on the engine block in the wake. The LAURA code compared well to ground based data at Mach 10 for heating levels due to free shear layer flow impingement on a sting

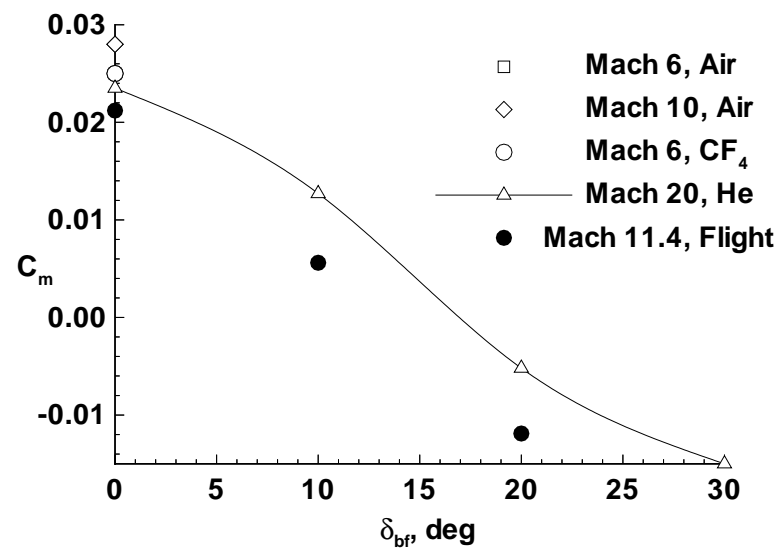

a) $C_{m}$

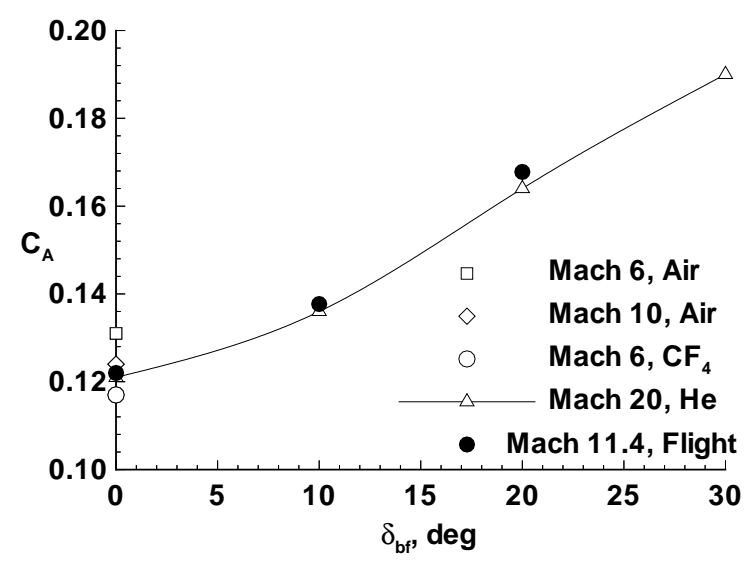

b) $C_{A}$

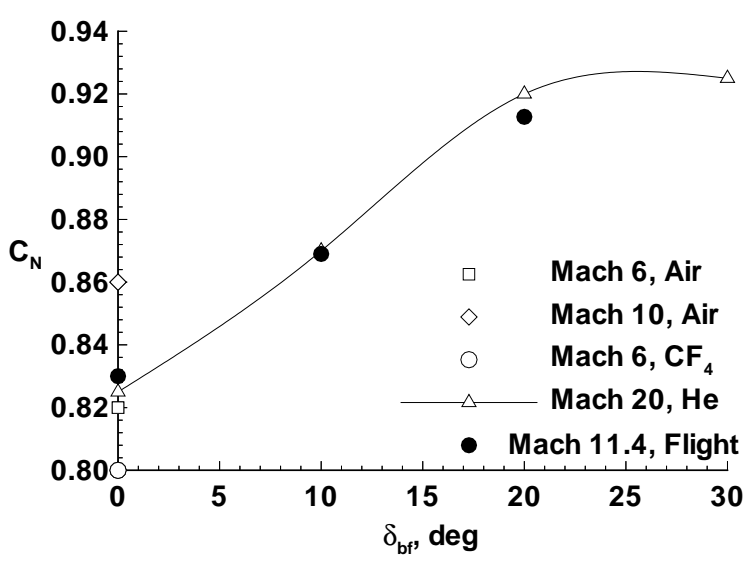

c) $C_{N}$

Fig. 18 Aerodynamic coefficients measured in ground based experiments and predicted for the flight environment as function of bodyflap deflection angle for B1001D configuration at $\alpha=36 \mathrm{deg}$. 
in the wake of a blunt body using laminar, steady flow assumptions. ${ }^{136}$ However, transitional effects have a strong influence on impingement heating levels in the base region and are underpredicted using laminar flow assumptions. ${ }^{169}$ Unlike the bodyflap solution from Phase I work, the present wake flow solutions showed no indication of unsteady flow. Two-equation turbulence models are currently being evaluated for application to this problem.

\section{Leveraging}

Leveraging refers to the use of engineering approximations, ${ }^{170}$ often based on boundary-layer methods, to extend (leverage) a limited matrix of CFD solutions for better coverage of the parameter space.

Recent experiences with the Phase I design process for X-33 have revealed opportunities for development of software to better exploit a limited CFD solution matrix. Extraction of only a few parameters at the surface and at the boundary-layer edge of a CFD solution can enable analytic extension of heat transfer solutions beyond the baseline matrix. The approach (defined as Method $1 \mathrm{G}^{171,172}$ ) extracts CFD derived quantities at the wall and at the boundary layer edge for inclusion in a post-processing boundary-layer analysis. It allows a designer at a workstation to address two questions, given a single CFD solution. (1) How much does the heating change for a thermal protection system with different catalytic properties than was used in the original CFD solution? (2) How does the heating change at the interface of two different TPS materials with an abrupt change in catalytic efficiency? The answer to the second question is particularly important, because abrupt changes from low to high catalytic efficiency can lead to localized increase in heating which exceeds the usually conservative estimate provided by a fully catalytic wall assumption. Design iterations are conducted without need of additional CFD runs until convergence on a single concept is achieved, at which point CFD could be used to provide a final check and/or recalibration point. Perhaps more importantly, it allows the design team to assess the effect of changes in some material properties heating rate without the need to rerun archived solutions.

The front section of the RLV configuration for a flowfield simulation at Mach 25, 45 deg. angle of attack, and $79.6 \mathrm{~km}$ altitude is examined. Heating rates for both a fully catalytic wall $\left(\gamma_{s}=1\right.$ in Eq. 5) and a finite-catalytic wall were computed at the respective radiative equilibrium wall temperatures. Circumferential heating distributions as a function of computational coordinate $j$ varying from leeside $(j=1)$ to windside $(j=64)$ are presented in Fig. 19a for the front section. The circumferential cut is from the $i=40$ plane which lies far downstream from the nose and upstream of a wing. The windside centerline heating distribution as a function of computational co-

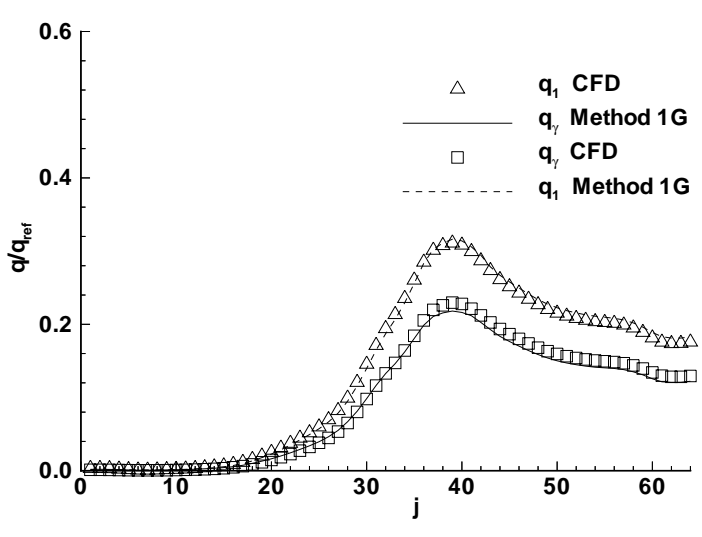

a) $i=40$, circumferential distribution

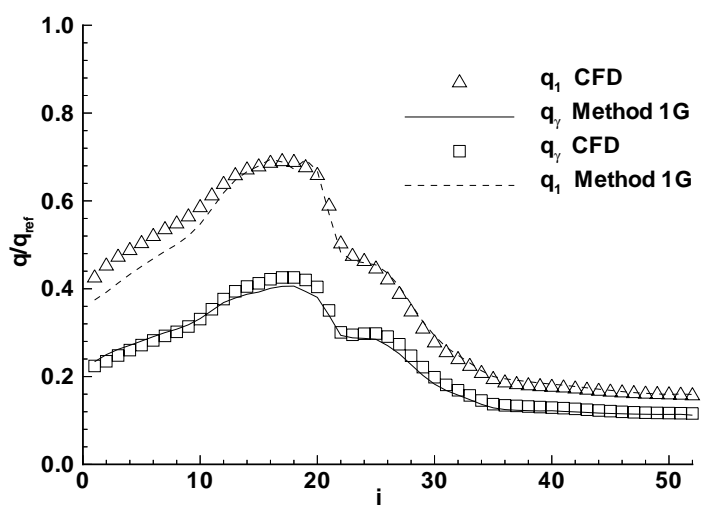

b) $j=64$, centerline distribution

Fig. 19 Comparison of CFD heating levels obtained on front section of RLV with Method 1G predictions obtained at $\gamma=\gamma(T)$ and $\gamma=1$ at Mach 25 and $79.6 \mathrm{~km}$.

ordinate $i$ varying from the stagnation point $(i=16)$ to the exit plane of the front section $(i=52)$ is presented in Fig. 19b. Integral boundary-layer corrections to CFD baseline heating are generally within $5 \%$ of computations. Even in the case where significant turning of streamlines occurs for flow expanding around the side of the vehicle $(32<j<48)$, in Fig. 19a the integral-boundary-layer extrapolation from the baseline CFD computation is an excellent predictor of the CFD result at the off-baseline catalysis model.

Consistency of these predictions over a broader range of entry conditions and geometric complexity remains to be established before integral boundary-layer methods (or a related approach) can be used with confidence in a design mode.

Boundary-layer codes may extract pressure and velocity fields from inviscid solutions over complex configurations to obtain heating distributions. Many more solutions per design cycle can be generated with this approach (when it is applicable) than with PNS or TLNS simulations. The Langley Approximate Three- 


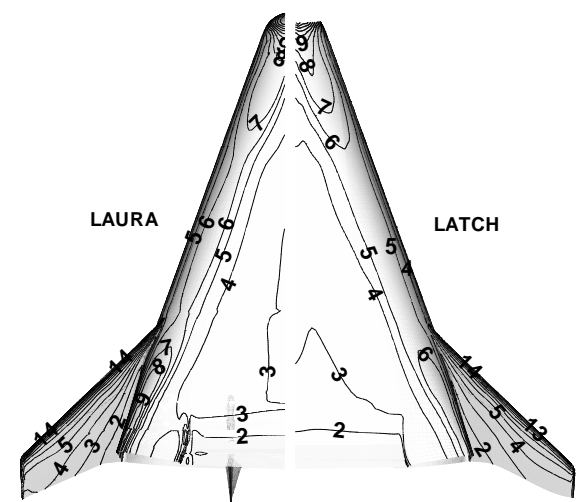

Fig. 20 Comparison of DPLUR/LATCH and LAURA laminar heating in $B T U / \mathrm{ft}^{2}-\mathrm{s}$ on $\mathrm{X}-33$ at Mach 11.47 and $\alpha=36.2 \mathrm{deg}$.

dimensional Convective Heating (LATCH) ${ }^{173}$ code has been extensively applied to X-33 and X-34 heating analyses using inviscid solutions predominantly from DPLUR but also from LAURA and FELISA. Interaction with FELISA is complicated at present by the requirement to interpolate unstructured grid results into the single-block, structured format required by LATCH. LATCH combines an axisymmetric analog for solution of the general three-dimensional boundary-layer equations in a generalized coordinate system. Inviscid surface streamlines and pressures are interpolated from inviscid solutions; streamline redistribution is used to obtain uniform coverage of complex surfaces. The method developed by Zoby et. $\mathrm{al}^{174}$ provides a fast, approximate method for solving the boundary-layer equations which has been shown to yield accurate results (within $\pm 10 \%$ of experimental data) for both wind-tunnel and conditions. LATCH is currently restricted to single-block, perfect-gas or equilibrium-gas applications. Laminar or turbulent solutions can be generated. While it does not yet handle the physical complexity of thermochemical nonequilibrium as in codes like the Boundary Layer Integral Matrix Procedure (BLIMP) $)^{175}$ it is applicable to geometrically complex flows.

A sample application of LATCH to X-33 heating analysis is shown in Fig. 20 and compared to LAURA results. Agreement is generally very good. Even heating rates on the wing leading edges, where threedimensional effects are exceptionally strong, are within $20 \%$ of the LAURA results. The LATCH solution runs in minutes on a workstation. The inviscid DPLUR solution runs approximately four times faster than the viscous LAURA solution.

\section{Future}

The ongoing industry led design effort for X-33 has had a profound effect on the development and application of computational aerothermodynamic tools within NASA. The principle drivers in this environment have been: (1) the need to respond to evolving configurations with time consuming gridding requirements; (2) the need to produce and evaluate matrices of solutions in a tight time frame; and (3) the need to extract, interpret, and transfer appropriate subsets of the solutions in formats that are usable by other members of the design team. These drivers have been most valuable in understanding the capabilities and limitations of the computational tools that have been developed over the past decade.

Perhaps nothing is more inspiring or motivating to a code developer than daily confrontation with inadequate software and demanding timelines. In some cases, software modifications required to improve the work environment are simple and incremental. For example, several utilities (STRIDE, ENSEMBLE, BLOX, GRIDSWAP) have evolved in LAURA to facilitate grid sequencing, solution sequencing, block marching, component isolation, and volume grid restructuring to address $\mathrm{X}-33$ requirements. More flexible interblock boundary conditions and routines that apply integral boundary-layer heating analyses to CFD solutions were also specifically developed in LAURA for X-33. Many other needs are also easily identified but not so easily satisfied. As noted previously, slow convergence in the windside boundary layer and in separated regions preceding deflected bodyflaps can be partially overcome using block marching, component isolation, and physically motivated load balancing. However, recent advances (predominantly academic in nature) suggest that more substantial improvements could be achieved with new multigrid methods and pre-conditioning. Incorporating these algorithms in a flow environment with strong shocks and thermochemical source terms involves research and verification that are difficult to implement in the midst of an intense vehicle design phase. Similar comments can be made regarding grid generation issues and unstructured, Navier-Stokes flow solvers.

Current projects must proceed using the best tools on hand. Future projects will require better tools if we are to maintain a competitive edge. The challenge is to maintain a balance among research and development and application activities to serve the present and the future. Some thoughts on future needs specifically related to computational aerothermodynamics follow.

\section{Architectures and Algorithms}

CFD underwent a revolutionary change when computer architectures moved from serial to vector processing. Current computational techniques are based on traditional vector processor architectures that have not materially changed in the past 20 years. Although some effort is under way to port existing codes to a parallel environment, there is little work being done to exploit the full potential of these systems for hypersonic CFD. It is generally acknowledged that future 
increases in computing speed will come through the maturation of parallel processing systems. Most current systems are on the $(\mathrm{O}) 100$ processors and it is not difficult, using current solution algorithms and block domain decomposition strategies, to bring the full power of one of these systems to bear on a single problem. However, is this current approach rational or even applicable when these systems are (O)1000 or (O)10000 processors. We need to be looking now for new and innovative ways to take advantage of these systems.

\section{Convergence Acceleration}

Methods to simplify systems of governing equations by identifying partial equilibrium relations via computational singular perturbation could be explored. These ideas have been demonstrated in complex, thermodynamic systems and may have application in more conventional fluid mechanics situations. The techniques fundamentally deal with the question, "Why do some parts of the solution or some areas of the flow domain converge more quickly than others? Can we exploit this behavior to accelerate overall convergence?"

Preconditioning applied to the relaxation process offers similar potential for improvement, particularly in three-dimensional stagnation regions behind strong shocks and in the near-wall boundary layer which are characterized by near-zero eigenvalues in the Jacobian.

Application of Multigrid methods for hypersonic flows improves convergence times, but appears to fall far short of theoretical expectations. We typically see a factor of approximately three in speedup at the expense of additional memory overhead. Can we get large speedups on complex problems with thermochemical nonequilibrium gas chemistry? Are similar approaches possible that are not hindered by presence of strong shocks or strong source terms? What savings may be associated in applying specialized algorithms to strongly elliptic versus strongly hyperbolic or predominantly viscous versus predominantly inviscid flow domains? What are the best ways to exploit parallel computing and implement load balancing for these applications? Can asynchronous relaxation of various flow domains and/or equation sets be exploited for additional convergence acceleration?

\section{Physical Modeling}

Advances or new approaches in physical modeling are sought which offer improved computational efficiency. These include models for turbulent flow, transition from laminar to turbulent flow, energy exchange mechanisms, nonequilibrium kinetics, and radiation. Can we add sufficient "intelligence" to the algorithm to add or delete elements of the physical models on the fly where appropriate to reduce computation time while maintaining a physically correct simulation? Some aspects of physical modeling introduce new complexities in the equation sets. For example, radiative energy transport changes our system of PDE's to an integrodifferential equation system. The numerical solution of these sets generally employs loosely coupled relaxation algorithms that require three to five sequential passes between equation sets. How can we do it better?

Inclusion of all significant plasmadynamic effects in both weak and strongly ionized flows requires further effort. There is evidence ${ }^{176}$ that local sound speed and possibly drag are affected by low levels of externally produced ionization in ways which are not fully understood or predicted with the current state of physical models.

\section{Surface Definition and Grid Definition}

We need to be able to quickly define configurations. We need to be able to quickly alter configurations. We need a platform to execute these definitions and alterations that is simple (intuitive) to use. Analytic definitions (even very complex, patched ones) are preferable because they allow for various optimization studies. The platform (CAD system) should contain a library of shapes, appendages, configurations that can be quickly called up and assembled. Operations to rescale, twist, bend, stretch elements and automatically define intersections of elements as they are reconfigured should be available. Surface discretization should be carried along as part of this process. (If a wing is twisted, the associated surface grid should follow the deformation. If a wing is translated along a fuselage, the surface grid intersection points on both elements should be coordinated. The preservation of this information (orientation) is important to facilitate restart solutions.) Structured grid patches or full unstructured grid surface disretizations should be allowed. Automatic filleting of element intersections should be accommodated. New elements should be input via stereoscopic imaging, discrete data file, or analytic definition. Methods for volume grid generation/adaption must be advanced in parallel. Volume grid generation (structured and unstructured) takes too long (hours to weeks) even in cases when volume grids over similar configurations already exist.

In the comments which preceded, it was assumed that flow simulation requires discretization of the flow domain. Generally, this domain includes the outer mold line of the vehicle, a far field boundary, and the space in between. Multi-Disciplinary Optimization (MDO) analyses including effects of response of the vehicle structure to aerodynamic and thermal loads (flexing, ablation) are assumed to require additional discretization of the structure. At present, there are no known methods of analysis that do not require discretization. Numerical methods which start with an analytic surface definition (likely a very complex, patched set of definitions) that generate their own surface and volume discretizations on the fly should 
be investigated. Possibilities for obtaining analytic or semianalytic solutions to augment the simulations and reduce or eliminate the need for volume discretization should be explored (keeping in mind we do not want to sacrifice ability to treat complex configurations).

Gridding strategies in the future will likely exploit overset grids and/or a combination of structured and unstructured. The state-of-the-art for unstructured grid generation on complex configurations is judged to be superior to structured grid capability using the simple metric of time to generate a complete grid. Once one has the complexity of unstructured algorithm available is there still advantage to maintaining structured formulation? Preliminary results with codes like DPLUR ${ }^{142}$ indicate some performance advantages with structured grid formulations on SIMD machines. If truly multidimensional viscous and inviscid capabilities are present, unstructured approaches may be preferable.

\section{Acknowledgements}

Kay Wurster of VAB/SASPG/LaRC performed the MINIVER analyses. Norma Bean of Computer Sciences Corp. (GeoLab) performed the surface grid generation for X33 and RLV on configurations defined by Lockheed. Steve Alter of Lockheed (Hampton) generated volume grids for X33 and RLV. Karen Bibb (AB/AGDD/RTG/LaRC) reviewed material on FELISA and supplied Fig. 12. Matt Rhode (AB/AGDD/RTG/LaRC) supplied previously unpublished experimental data on COMET/METEOR aerodynamics. Bill Scallion (AB/AGDD/RTG/LaRC) supplied previously unpublished experimental data on X-33 aerodynamics. Bobby Braun (VAB/SASPG/LaRC) helped with Fig. 1 and the discussion of the aeroshell design process.

\section{References}

${ }^{1}$ Cook, S. A., "X-33 Reusable Launch Vehicle Structural Technologies," AIAA 96-4573, AIAA, November 1996.

${ }^{2}$ Walberg, G. D., "A Survey of Aeroassisted Orbit Transfer," Journal of Spacecraft and Rockets, Vol. 22, No. 1, JanuaryFebruary 1985, pp. 3-18.

${ }^{3}$ Arrington, J. P. and Jones, J. J., editors, Shuttle Performance: Lessons Learned, Part I and II, March 1983, Conference Publication 2283.

${ }^{4}$ Chamberlain, R. R., "Calculation of Three-Dimensional Jet Interaction Flowfields," AIAA 90-2099, AIAA, July 1990.

${ }^{5}$ Mendenhall, M. R., Lesieutre, D. J., Caruso, S. C., Dillenius, M. F. E., and Kuhn, G. D., "Aerodynamic Design of Pegasus," AIAA 91-0190, AIAA, January 1991.

${ }^{6}$ McClinton, C., Bittner, R., and Kamath, P., "CFD Support of NASP Design," AIAA 90-5249, AIAA, October 1990.

${ }^{7}$ Grassin, C., "Computation of Aerodynamic Coefficients on Hermes - Ariane5 Configuration," Aerothermodynamics for Space Vehicles, Proceedings of the First European Symposium ESTEC, May 1991, pp. 537-542.

${ }^{8}$ Hirschel, E. H., "Aerothermodynamic Challenges of the SANGER Space Transportation System," Aerothermodynamics for Space Vehicles, Proceedings of the First European Symposium ESTEC, May 1991, pp. 549-558.

${ }^{9}$ Burns, B. R. A., "Aerodynamic Design Challenges of a Single Stage to Orbit, Reusable Launch Vehicle," International Conference on Hypersonic Aerodynamics, The Royal Aeronautical Society, September 1989, pp. 1.1-1.27.

${ }^{10}$ Velazquez, A., Huot, J.-P., and Molina, R.-C., "Aerothermodynamics Effects During the Huygens Entry Phase," Aerothermodynamics for Space Vehicles, Proceedings of the First European Symposium ESTEC, May 1991, pp. 61-66.

${ }^{11}$ Neiland, V. Y., "Aerothermodynamic Configuration of First-Generation Aerospace Planes (of "BURAN"-Type) and First Flight Results," Aerothermodynamics for Space Vehicles, Proceedings of the First European Symposium ESTEC, May 1991, pp. 13-22.

${ }^{12}$ Levine, J., "NASA X-34 Program," Meeting Papers on Disc A9710806, AIAA, November 1996.

${ }^{13}$ Campbell, C. H., Caram, J. M., Li, C. P., and Madden, C. B., "Aerothermodynamic Environment Definition For an X23/X-24A Derived Assured Crew Return Vehicle," AIAA 961862, AIAA, June 1996.

${ }^{14}$ Hicks, J. W. and Trippensee, G., "NASA Hypersonic XPlane Flight Development of Technologies and Capabilities for the 21st Century Access to Space," Future AerospaceTechnology in Service to the Alliance C-32, AGARD, April 1997.

${ }^{15}$ Wessling, F. C., Robinson, M., Martinez, R. S., Gallimore, T., and Combs, N., "Commercial Experiment Transporter COMET," Journal of Spacecraft and Rockets, Vol. 31, No. 5, September-October 1994, pp. 846-854.

${ }^{16}$ Shirley, D. L. and McCleese, D. J., "Mars Exploration Program Strategy - 1995-2020," AIAA 96-0333, AIAA, January 1996.

${ }^{17}$ Fullmer, R. R., "Dynamic Ground Testing of the Skipper Attitude Control System," AIAA 96-1013, AIAA, January 1996.

${ }^{18}$ Richards, W. L. and Monaghan, R. C., "Design of a Flight Article for a Mach-8 Boundary-Layer Experiment," AIAA 964565, AIAA, November 1996.

${ }^{19}$ Kordulla, W. and Morice, P., "Computational Simulation of Hypersonic External Flow - Status of CFD in Europe," Future AerospaceTechnology in Service to the Alliance C-14, AGARD, April 1997.

${ }^{20}$ Maita, M. and Kobayashi, S., "Japanese Spaceplane and Hypersonic Technology Program Overiew - Progress of 19951996," Meeting Papers on Disc A9710804, AIAA, November 1996.

${ }^{21}$ Yamamoto, Y., Wada, Y., and Yoshioka, M., "Hypersonic CFD Analysis for the Aerothermodynamic Design of HOPE," AIAA 95-1770, AIAA, June 1995.

${ }^{22}$ Yamamoto, Y. and Yoshioka, M., "CFD and FEM Coupling Analysis of OREX Aerothermodynamic Flight Data," AIAA 95-2087, AIAA, June 1995.

${ }^{23}$ Gupta, R. N., Moss, J. N., and Price, J. M., "Assessment of Thermochemical Nonequilibrium and Slip Effects for Orbital Reentry Experiment (OREX)," AIAA 96-1859, AIAA, June 1996.

${ }^{24}$ Shanks, S. P., Srinivasan, G. R., and Nicolet, W. E., "AFWAL Parabolized Navier-Stokes Code: Formulation and Users Manual," TR 82-3034, AFWAL, June 1982.

${ }^{25}$ Moss, J. N., Anderson, E. C., and Bolz, C. W., "ViscousShock-Layer Solutions with Radiation and Ablation Injection for Jovian Entry," AIAA 75-0671, AIAA, May 1975.

${ }^{26}$ Golovachov, Y. P., Numerical Simulation of Viscous Shock Layer Flows, Kluwer Academic Publishers, 1995.

${ }^{27}$ Chen, Y. K., Henline, W. D., and Tauber, M. E., "Trajectory Based Heating and Ablation Calculation for Mars Pathfinder Aeroshell," Journal of Spacecraft and Rockets, Vol. 32, No. 2, March 1995, pp. 225-230.

${ }^{28}$ van Leer, B., "Towards the Ultimate Conservation Scheme: A Second-Order Sequel to Gudonov's Method," Journal of Computational Physics, Vol. 32, 1979, pp. 101-136. 
${ }^{29}$ Roe, P. L., "Approximate Riemann Solvers, Parameter Vectors, and Difference Schemes," Journal of Computational Physics, Vol. 43, October 1981, pp. 357-372.

${ }^{30}$ Harten, A., "High Resolution Schemes for Hyperbolic Conservation Laws," Journal of Computational Physics, Vol. 49, No. 2, February 1983, pp. 357-393.

${ }^{31}$ Swanson, R. C., Radespiel, R., and Turkel, E., "On Some Numerical Dissipation Schemes," AIAA 97-1945, AIAA, JuneJuly 1997

${ }^{32}$ Palmer, G., "A Comparison of Three-Dimensional Nonequilibrium Solution Algorithms Applied to Hypersonic Flows with Stiff Chemical Source Terms," AIAA 93-2861, AIAA, July 1993 .

${ }^{33}$ Gnoffo, P. A., "Point-Implicit Relaxation Strategies for Viscous, Hypersonic Flows," Computational Methods in Hypersonic Aerodynamics, edited by T. K. S. Murthy, Computational Mechanics Publications, Kluwer Academic Publishers, 1991, pp. $115-151$.

${ }^{34}$ Yoon, S. and Jameson, A., "Lower-Upper SymmetricGauss-Seidel Method for the Euler and Navier-Stokes Equations," AIAA Journal, Vol. 26, No. 9, September 1988, pp. $1025-1026$

${ }^{35}$ Eberhardt, S. and Imlay, S., "A Diagonal Implicit Scheme for Computing Flows with Finite-Rate Chemistry," AIAA 901577, AIAA, June 1990.

${ }^{36}$ Olynick, D. R., A New LU-SGS Flow Solver for Calculating Reentry Flows, Ph.D. thesis, North Carolina State University, 1992.

${ }^{37}$ Shuen, J.-S., "Upwind Differencing and LU Factorization for Chemical Nonequilibrium Navier-Stokes Equations," Journal of Computational Physics, Vol. 99, No. 2, 1992, pp. 233-250.

${ }^{38} \mathrm{Li}$, C.-P., "Computational Aspects of Chemically Reacting Flows " AIAA 91-1574-CP, AIAA, June 1991.

${ }^{39}$ Shuen, J.-S. and Yoon, S., "Numerical Study of Chemically Reacting Flows Using Lower-Upper Symmetric Successive Over-relaxation Scheme," AIAA Journal, Vol. 27, No. 12, 1989, pp. $1752-1760$.

${ }^{40}$ Lin, H. and Chieng, C.-C., "An Efficient Preconditioning Bi-CGSTAB Algorithm to Compute Flows in Chemical Nonequilibrium," AIAA 96-0774, AIAA, January 1996.

${ }^{41}$ Pulliam, T. H., "Time Accuracy and the Use of Implicit Methods," AIAA 93-3360-CP, AIAA, July 1993.

${ }^{42}$ Yee, H. C. and Sweby, P. K., "Nonlinear Dynamics \& Numerical Uncertainties in CFD," TM 110398, NASA, 1996.

${ }^{43}$ Edwards, J. R., "An Implicit Multigrid Algorithm for Computing Hypersonic, Chemically Reacting Viscous Flows," Journal of Computational Physics, Vol. 123, 1996, pp. 84-95.

${ }^{44}$ Greene, F. A., "Application of the Multigrid Solution Technique to Hypersonic Entry Vehicles," Journal of Spacecraft and Rockets, Vol. 31, September-October 1994, pp. 744-750.

${ }^{45}$ Radespiel, R. and Swanson, R. C., "Progress with Multigrid Schemes for Hypersonic Flow Problems," CR 189579, NASA, December 1991

${ }^{46}$ Lee, D. and van Leer, B., "Progress in Local Preconditioning of the Euler Navier-Stokes Equations," AIAA 93-3328, AIAA, July 1993.

${ }^{47}$ Lam, S. H., "Final Report on Computational Reacting Gas Dynamics," Final Grant Report NAG-1-726, NASA, January 1993, also available from Princeton University.

${ }^{48}$ Goussis, D., Lam, S., and Gnoffo, P., "Reduced and Simplified Chemical Kinetics for Air-Dissociation Using Computational Singular Perturbation," AIAA 90-0644, AIAA, January 1990.

${ }^{49}$ Gnoffo, P. A., Weilmuenster, K. J., and Alter, S. J., "Multiblock Analysis for Shuttle Orbiter Re-entry Heating from Mach 24 to Mach 12," Journal of Spacecraft and Rockets, Vol. 31, No. 3, May-June 1994, pp. 367-377.

${ }^{50}$ Gnoffo, P. A., "Hypersonic Flows Over Biconics Using a Variable-Effective-Gamma, Parabolized-Navier-Stokes Code," AIAA 83-1666, AIAA, July 1983.
${ }^{51}$ Gnoffo, P. A., Weilmuenster, K. J., Braun, R. D., and Cruz, C. I., "Influence of Sonic-Line Location on Mars Pathfinder Probe Aerothermodynamics," Journal of Spacecraft and Rockets, Vol. 33, No. 2, March-April 1996, pp. 169-177.

${ }^{52}$ Prabhu, R. K. and Erickson, W. D., "A Rapid method for the Computation of Equilibrium Chemical Composition of Air to $15000 \mathrm{~K}$," TP 2792, NASA, March 1988.

${ }^{53}$ Liu, Y. and Vinokur, M., "Equilibrium Gas Flow Computations. I. Accurate and Efficient Calculation of Equilibrium Gas Properties," AIAA 89-1736, AIAA, June 1989.

${ }^{54}$ Capitelli, M., Colonna, G., Gorse, C., and Giordano, D., "Survey of Methods of Calculating High-Temperature Thermodynamic Properties of Air Species," STR 236, European Space Agency, December 1994.

${ }^{55}$ Giordano, D., Capitelli, M., Colonna, G., and Gorse, C., "Tables of Internal Partition Functions and Thermodynamic Properties of High temperature Air Species from $50 \mathrm{~K}$ to 100000 K," STR 237, European Space Agency, December 1994.

${ }^{56}$ Srinivasan, S., Tannehill, J. C., and Weilmuenster, K. J., "Simplified Curve Fits for the Thermodynamic Properties of Equilibrium Air," RP 1181, NASA, August 1987.

${ }^{57}$ Sutton, K., "Relations for the Thermodynamics and Transport properties in the Testing Environment of the Langley Hypersonic CF4 Tunnel," TM 83220, NASA, 1981.

${ }^{58}$ Park, C., "Review of Chemical-Kinetic Problems of Future NASA Missions, I: Earth Entries," Journal of Thermphysics and Heat Transfer, Vol. 7, No. 3, July-September 1993, pp. 385-398.

${ }^{59}$ Park, C., Howe, J. T., Jaffe, R. L., and Candler, G. V., "Review of Chemical-Kinetic Problems of Future NASA Missions, II: Mars Entries," Journal of Thermphysics and Heat Transfer, Vol. 8, No. 1, January-March 1994, pp. 9-23.

${ }^{60}$ Bose, D. and Candler, G. V., "Simulation of Hypersonic Flows Using a Detailed Nitric Oxide Formation Model," AIAA 96-1801, AIAA, June 1996.

${ }^{61}$ Levin, D. A., Candler, G. V., Collins, R. J., Erdman, P. W., Zipf, E. C., and Howlett, C., "Examination of Theory for Bow Shock Ultraviolet Rocket Experiments-I," Journal of Thermophysics and Heat Transfer, Vol. 8, No. 3, May-June 1994, pp. 447-452.

${ }^{62}$ Levin, D. A., Braunstein, M., Candler, G. V., Collins, R. J., and Smith, G., "Examination of Theory for Bow Shock Ultraviolet Rocket Experiments-II," Journal of Thermophysics and Heat Transfer, Vol. 8, No. 3, May-June 1994, pp. 453-459.

${ }^{63}$ Macheret, S. O., Fridman, A. A., Adamovich, I. V., Rich, J. W., and Treanor, C. E., "Mechanisms of Nonequilibrium Dissociation of Diatomic Molecules," AIAA 94-1984, AIAA, 1994.

${ }^{64}$ Losev, S. A., "Two-Temperature Chemical Kinetics in Gas Dynamics," AIAA 96-2026, AIAA, June 1996.

${ }^{65}$ Kovach, E. A., Losev, S. A., and Sergievskaya, A. L., "Two-Temperature Kinetic Models for Dissociation of Molecules in Strong Shock Waves," Chem.Phys.Repts, Vol. 14, No. 9, 1995, pp. 1353-1387.

${ }^{66}$ Sergievskaya, A. L., Kovach, E. A., and Losev, S. A., Opit Informatsionno-Matematicheskovo Modelirovniya $v$ FizikoChimicheskoi Kinetike(Practice in Empirical-Mathematical Models for Physico-Chemical Kinetics), Moscow State University, 103009, Moscow, Gertsena St., 5/7, Institute of Mechanics, Moscow State University, 117192, Moscow, Michurinskii Pr., 1, 1995.

${ }^{67}$ Knab, O., Frühauf, H.-H., and Messerschmid, E. W., "Theory and Validation of the Physically Consistent VibrationChemistry-Vibration Model," Journal of Thermophysics and Heat Transfer, Vol. 9, No. 2, March-April 1995, pp. 219-226.

${ }^{68}$ McBride, B., Heimel, S., Ehlers, J. G., and Gordon, S., "Thermodynamic Properties to $6000 \mathrm{~K}$ for 210 Substances Involving the First 18 Elements," SP 3001, NASA, 1963.

${ }^{69}$ Olynick, D. R. and Henline, W. D., "Comparison of Coupled Radiative Flow Solutions with Project Fire II Flight Data," 
Journal of Spacecraft and Rockets, Vol. 9, No. 4, OctoberDecember 1995, pp. 586-594.

${ }^{70}$ Macheret, S. O. and Rich, J. W., "Nonequilibrium Dissociation Rates Behind Strong Shock Waves: Classical Model," Chemical Physics, Vol. 174, 1993, pp. 25-43.

${ }^{71}$ Candler, G. V., Olejniczak, J., and Harrold, B., "Detailed Simulation of Nitrogen Dissociation in Stagnation Regions," AIAA 96-2025, AIAA, June 1996.

${ }^{72}$ Hirschfelder, J. O., Curtiss, C. F., and Bird, R. B., Molecular Theory of Gases and Liquids, John Wiley \& Sons, Inc., New York, 1954.

${ }^{73}$ Gupta, R. N., Lee, K.-P., Thompson, R. A., and Yos, J. M., "Calculations and Curve Fits of Thermodynamic and Transport Properties for Equilibrium Air to 30000 K," Reference Publication 1260, NASA, October 1991.

${ }^{74}$ Gupta, R. N., Yos, J. M., Thompson, R. A., and Lee, K.-P., "A Review of Reaction Rates and Thermodynamic and Transport Properties for an 11-Species Air Model for Chemical and Thermal Nonequilibrium Calculations to 30000 K," Reference Publication 1232, NASA, August 1990.

${ }^{75}$ Yos, J. M., "Transport Properties of Nitrogen, Oxygen, and Air to 30,000 K," Technical Memorandum RAD TM-63-7, AVCO-RAD, March 1963.

${ }^{76}$ Wilke, C. R., "A Viscosity Equation for Gas Mixtures," Journal of Chemical Physics, Vol. 18, No. 4, April 1950, pp. 517-519.

${ }^{77}$ Palmer, G., "An Assessment of Transport Property Methodologies for Hypersonic Flows," AIAA 97-0983, AIAA, January 1997.

${ }^{78}$ Curtiss, C. F. and Hirschfelder, J. O., "Transport Properties of Multicomponent Gas Mixtures," The Journal of Chemical Physics, Vol. 17, No. 6, June 1949, pp. 550-555.

${ }^{79}$ Olynick, D. R. and Henline, W. D., "Numerical Benchmarks for Navier-Stokes Heating Calculations on Access to Space Vehicles," AIAA 95-2078, AIAA, June 1995.

${ }^{80}$ Harpold, J. C. and Claude A. Grave, J., "Shuttle Entry Guidance," Journal of the Astronautical Sciences, Vol. 27, No. 3, July-September 1979, pp. 239-268.

${ }^{81}$ Tim, T. C. and Olynick, D. R., "An Investigation of Possible Shuttle Upgrades Using Advance TPS Concepts," AIAA 97-0277, AIAA, January 1997.

${ }^{82}$ Park, C., Nonequilibrium Hypersonic Aerothermodynamics, John Wiley \& Sons, Inc, New York, 1990.

${ }^{83}$ Stewart, D. A., Pallix, J. B., and Esfahani, L., "Surface Catalytic Efficiency of Candidate Ceramic Thermal Protection Materials for SSTO," CDTM 20007, NASA, March 1995.

${ }^{84}$ Stewart, D. A., "Determination of Surface Catalytic Efficiency for Thermal Protection Materials - Room Temperature to Their Upper Use Limit ," Tech. Rep. 96-1863, AIAA, June 1996.

${ }^{85}$ Milos, F. S. and Rasky, D. J., "Review of Numerical Procedures for Computational Surface Thermochemistry," Journal of Thermophysics and Heat Transfer, Vol. 8, No. 1, JanuaryMarch 1994, pp. 24-34.

${ }^{86}$ Milos, F. S. and Chen, Y. K., "Comprehensive Model for Multi-component Ablation Thermochemistry," AIAA 97-0141, AIAA, January 1997.

${ }^{87}$ Baldwin, B. S. and Lomax, H., "Thin Layer Approximation and Algebraic Model for Separated Turbulent Flows," AIAA 78-0257, AIAA, January 1978.

${ }^{88}$ Cebeci, T. and Smith, A. M. O., "A Finite-Difference Method for Calculating Compressible Laminar and Turbulent Boundary Layers," Journal of Basic Engineering, September 1970, pp. 523-535.

${ }^{89}$ Gupta, R. N., Lee, K. P., Zoby, E. V., Moss, J. N., and Thompson, R. A., "Hypersonic Viscous Shock-Layer Solutions Over Long Slender Bodies - Part I: High Reynolds Number Flows," Journal of Spacecraft and Rockets, Vol. 27, No. 2, March-April 1990, pp. 175-184.
${ }^{90}$ Goldberg, U. C., "Separated Flow Treatment with a New Turbulence Model," AIAA Journal, Vol. 24, No. 10, October 1986, pp. 1711-1713.

${ }^{91}$ Wilcox, D. C., Turbulence Modeling for CFD, DCW Industries, Inc., 5354 Palm Drive, La Cañada, California 91011, 1993.

${ }^{92}$ Kumar, A., Gnoffo, P. A., Moss, J. N., and Drummond, J. P., "Advances in Computational Capabilities for Hypersonic Flows," Future AerospaceTechnology in Service to the Alliance C-13, AGARD, April 1997.

${ }^{93}$ Malik, M. R. and Chang, C.-L., "Boundary-Layer Stability and Transition," HTC 9407, High Technology Corporation, 28 Research Dr., P.O. Box 7262, Hampton VA 23666, August 1994.

${ }^{94}$ Hussaini, M. Y., Kumar, A., and Streett, C. L., editors, Instability, Transition, and Turbulence, Springer Verlag, New York, 1992

${ }^{95}$ Godil, A. and Bertelrud, A., "Design of a Wing Shape for Study of Hypersonic Crossflow Transition in Flight," Computing Systems in Engineering, Vol. 3, No. 1-4, 1992, pp. 115-130.

${ }^{96}$ Bushnell, D. M., "Hypersonic Flight Experimentation Status and Shortfalls," Future AerospaceTechnology in Service to the Alliance C-29, AGARD, April 1997.

${ }^{97}$ Bouslog, S. A., An, M. Y., Hartmann, L. N., and Derry, S. M., "Review of Boundary-Layer Transition Flight Data on the Space Shuttle Orbiter," AIAA 91-0741, AIAA, January 1991.

${ }^{98}$ Tauber, M. E. and Sutton, K., "Stagnation-Point Radiative Heating Relations for Earth and Mars Entries," Journal of Spacecraft and Rockets, Vol. 28, No. 1, January-February 1991, pp. $40-42$.

${ }^{99}$ John D. Anderson, J., Hypersonic and High Temperature Gas Dynamics, McGraw-Hill, Inc., 1989.

${ }^{100}$ Cornette, E. S., "Forebody Temperatures and Calorimeter Heating Rates Measured During Project Fire II Reentry at 11.35 km/s ," TM X 1305, NASA, Nov. 1966.

${ }^{101}$ Park, C., "Nonequilibrium Air Radiation (NEQUAIR) Program: User's Manual," TM 86707, NASA, 1985.

${ }^{102}$ Chambers, L. H., "Predicting Radiative Heat Transfer in Thermochemical Nonequilibrium Flow Fields," TM 4564, NASA, Sept. 1994.

${ }^{103}$ Hartung, L. C. and Hassan, H. A., "Radiation Transport Around Axisymmetric Blunt Bodies Using a Modified Differential Approximation," Journal of Thermophysics and Heat Transfer, Vol. 7, No. 2, April-June 1993, pp. 220-227.

${ }^{104}$ Carlson, L. A. and Gally, T. A., "Nonequilibrium Chemical Radiation and Coupling, Part 1: Theory and Models," Journal of Thermophysics and Heat Transfer, Vol. 6, No. 3, July-September 1992, pp. 385-391.

${ }^{105}$ Gupta, R. N., Kam-Pui, L., Moss, J. N., and Sutton, K., "Viscous-Shock-Layer Solutions with Coupled Radiation and Ablation Injection for Earth Entry ", AIAA 90-1697, AIAA, June 1990.

${ }^{106}$ Nicolet, W. E., "Advanced Methods for Calculating Radiation Transport Ablation-Product Contaminated Boundary Layers," CR 1656, NASA, 1970.

${ }^{107}$ Milos, F. S., "Galileo Probe Heat Shield Ablation Experiment ," AIAA 96-1823, June 1996.

${ }^{108}$ Bird, G. A., Molecular Gas Dynamics, Clarendon Press, Oxford, 1976.

${ }^{109}$ Gilmore, M. R., Gallis, M. A., and Harveyr, J. K., "Breakdown of Continuum Solvers in Rapidly Expanding Flows," AIAA 95-2134, AIAA, June 1995.

${ }^{110}$ Gupta, R. N., Scott, C. D., and Moss, J. N., "SlipBoundary Equations for Multicomponent Nonequilibrium Airflow," TP 2452, NASA, November 1985.

${ }^{111}$ Gupta, R. N. and Simmonds, A. L., "Hypersonic LowDensity Solutions of the Navier-Stokes Equations with Chemical Nonequilibrium and Multicomponent Surface Slip," AIAA 861349, AIAA, June 1986. 
${ }^{112}$ Wood, W. A., Gnoffo, P. A., and Rault, D. F. G., "Aerodynamic Analysis of Commercial Experiment Transporter ReEntry Capsule," Journal of Spacecraft and Rockets, Vol. 33, No. 5, September-October 1996, pp. 643-646.

${ }^{113}$ Blake, M. W., Kerr, P. A., Thorpe, S. A., and Chou, J. J., "NASA Geometry Data Exchange Specification for Computational Fluid Dynamics(NASA IGES)," RP 1338, NASA, April 1994.

${ }^{114}$ Wulf, A. and Akdag, V., "Tuned Grid Generation With ICEM CFD," CP 3291, NASA, May 1995

${ }^{115}$ Steinbrenner, J., Chawner, J., and Pouts, C., "Multiple Block Grid Generation in the Interactive Environment," AIAA 90-1602, AIAA, June 1990.

${ }^{116}$ Gaither, A. and et. al., "The National Grid Project: A System Overview," CP 3291, NASA, May 1995.

${ }^{117}$ Soni, B. K., "A User's Guide To GENIE," Tech. rep., Mississippi State University, November 1991.

${ }^{118}$ Thompson, J. F., "A Composite Grid Generation Code For General 3D Regions - the EAGLE code," AIAA, Vol. 26, March 1988, pp. 1-10.

119 Sorenson, R., "The 3DGRAPE BOOK: Theory, User's Manual, Examples," TM 102224, NASA, May 1993.

${ }^{120}$ Alter, S. J. and Weilmuenster, K. J., "The ThreeDimensional Multi-Block Advanced Grid Generation System," TM 108985, NASA, May 1993.

${ }^{121}$ Sorenson, R. L. and Alter, S. J., "3DGRAPE/AL: The Ames/Langley Technology Upgrade," CP 3291, NASA, May 1995

122 Alter, S. J., "The Volume Grid Manipulator (VGM): A Grid Reuseability Tool," CR 4772, NASA, April 1997.

${ }^{123}$ Davies, C. B. and Venkatapathy, E., "The multidimensional self-adaptive grid code, SAGEv2," TM 110350, NASA, 1995

${ }^{124}$ Naim, A., Mallet, M., Rostand, P., and Hasholder, J. M., "Local Aerothermal Problems During Hermes Reentry," CP 514, AGARD, 1992, Paper No. 42.

${ }^{125}$ Peiro, J., Peraire, J., and Morgan, K., "FELISA System Reference Manual and User's Guide," Technical report, University College Swansea, 1993.

${ }^{126}$ Frink, N. T., Pirzadeh, S., and Parikh, P., "Grid Software System For Solving Complex Aerodynamic Problems," CP 3291, NASA, May 1995.

${ }^{127}$ Aftosmis, M. J., Berger, M. J., and Melton, J. E., "Robust and Efficient Cartesian Mesh Generation for Component-Based Geometry," AIAA 97-0196, AIAA, 1997.

${ }^{128}$ Mehta, U., "The Aerospace Plane Design Challenge: Credible Computational Fluid Dynamics Results," AIAA 905248, AIAA, October 1990.

${ }^{129}$ Roache, P. J., "Need for Control of Numerical Accuracy," Journal of Spacecraft and Rockets, Vol. 27, No. 2, March-April 1990 .

${ }^{130}$ Holden, M. S., Moselle, J. R., Sweet, S. J., and Martin, S. C., "A Database of Aerothermal Measurements in Hypersonic Flow for CFD Validation," AIAA 96-4597, AIAA, November 1996.

${ }^{131}$ Holden, M. S. and Walker, B. J., "A Review of Recent Development, Testing and Code Validation Studies in the LENS Facility," AIAA 96-4598, AIAA, November 1996.

132 Olejniczak, J., Candler, G. V., Wright, M. J., Hornung, H. G., and Leyva, I. A., "High Enthalpy Double-Wedge Experiments," AIAA 96-2238, AIAA, June 1996.

${ }^{133}$ Yee, H. C., "On Symmetric and Upwind TVD Schemes," TM 88325, NASA, 1986.

${ }^{134}$ Cheatwood, F. M. and Gnoffo, P. A., "User's Manual for the Langley Aerothermodynamic Upwind Relaxation Algorithm (LAURA)," TM 4674, NASA, April 1996.

${ }^{135}$ Weilmuenster, K. J., Gnoffo, P. A., and Greene, F. A., "Navier-Stokes Simulations of Orbiter Aerodynamic Character- istics Including Pitch Trim and Bodyflap," Journal of Spacecraft and Rockets, Vol. 31, No. 3, May-June 1994, pp. 355-366.

${ }^{136}$ Gnoffo, P. A., "Code Calibration Program in Support of the Aeroassist Flight Experiment," Journal of Spacecraft and Rockets, Vol. 27, No. 2, March-April 1990, pp. 131-142.

${ }^{137}$ Rudy, D. H., Thomas, J. L., Kumar, A., Gnoffo, P. A. and Chakravarthy, S. R., "Computation of Laminar Hypersonic Compression-Corner Flows," AIAA Journal, Vol. 29, No. 7, July 1991, pp. 1108-1113.

${ }^{138}$ Saad, Y. and Schultz, M., "GMRES: A Generalized Minimal Residual Algorithm for Solving Nonsymmetric Linear Systems," SIAM Journal of Scientific and Statistical Computing, Vol. 7, No. 3, 1986, pp. 856-869.

139 AeroSoft, I., GASP Version 3 User's Manual, AeroSoft, Inc., 1872 Pratt Drive, Suite 1275, Blacksburg, VA 24060-6363, May 1996.

${ }^{140}$ Olynick, D. and Tam, T., "Trajectory based validation of the Shuttle Heating Environment," AIAA 96-1891, AIAA, June 1996.

${ }^{141}$ Candler, G. V. and MacCormack, R. W., "The Computation of HypersonicIonized Flows in Chemical and Thermal Nonequilibrium," Journal of Thermophysics and Heat Transfer, Vol. 5, No. 3, May-June 1991, pp. 266-273.

142 Candler, G. V., Wright, M. J., and McDonald, J. D., " Data-Parallel Lower-Upper Relaxation Method for Reacting Flows," AIAA Journal, Vol. 32, No. 12, December 1994, pp. $2380-2386$

${ }^{143}$ Bibb, K. L., Peraire, J., and Riley, C. J., "Hypersonic Flow Computations on Unstructured Meshes," AIAA 97-0625, AIAA, January 1997.

${ }^{144}$ Arminjon, P. and Dervieux, A., "Construction of TVDlike Artificial Viscosities on Two-Dimensional Arbitrary FEM Grids," Tech. Rep. Report 1111, INRIA, 1989

${ }^{145}$ Jameson, A., Reuther, J., and Martinelli, L., "Computational Algorithms for Aerodynamic Analysis and Design TLSP: Annual Report, 1 Oct 1993 - 30 Sept. 1994," AFOSR 95$0082 T R$, Princeton University, 1995.

${ }^{146}$ Reuter, W., Buning, P., and Hobson, G., "Flowfield Computations Over the Space Shuttle Orbiter with a Proposed Canard at a Mach Number of 5.8 and 50 Degrees Angle of Attack," AIAA 93-0322, AIAA, January 1993.

${ }^{147}$ Melton, J. E., Berger, M. J., Aftosmis, M. J., and Wong, M. D., "Development and Application of a 3D Cartesian Grid Euler Method," Surface Modeling, Grid Generation, and Related Issues in Computational Fluid Dynamic (CFD) Solutions, May 1995, NASA-CP-3291.

${ }^{148}$ Jameson, A., Schmidt, W., and Turkel, E., "Numerical Solutions of the Euler Equations by Finite Volume Methods Using Runge-Kutta Time-Stepping Schemes," AIAA 81-1259, AIAA, June 1981.

${ }^{149}$ Braun, R. D., Powell, R. W., Engelund, W. C., Gnoffo, P. A., Weilmuenster, K. J., and Mitcheltree, R. A., "Mars Pathfinder Six Degree-of-Freedom Entry Analysis," Journal of Spacecraft and Rockets, Vol. 32, No. 6, November-December 1995, pp. 993-1000.

${ }^{150}$ Olynick, D., Chen, Y.-K., and Tauber, M., "Forebody TPS Sizing with Radiation and Ablation for the Stardust Sample Return Capsule," AIAA 97-2474, AIAA, June 1997.

${ }^{151}$ Olynick, D., Chen, Y.-K., and Tauber, M., "Wake Flow Calculations with Radiation and Ablation for the Stardust Sample Return Capsule," AIAA 97-2477, AIAA, June 1997.

${ }^{152}$ Tran, H. K., Johnson, C. E., ta Hsu, M., Smith, M., Dill, H., and Chen-Johnson, A., "Qualification of the Forebody Heatshield of the Stardust Sample Return Capsule," AIAA 97-2482, AIAA, June 1997.

${ }^{153}$ Johnson, C. E., Tran, H. K., Smith, M., Dill, H., and Etienne, L., "Stardust Backshell and Back Interface Plate Design Verification Tests in the NASA Ames Arc Jet Facilities," AIAA 97-2483, AIAA, June 1997. 
${ }^{154}$ Mitcheltree, R. A., Wilmoth, R. G., Cheatwood, F. M., Braunckmann, J. G., and Greene, F. A., "Aerodynamics of Stardust Sample Return Capsule," AIAA 97-2304, AIAA, June 1997

${ }^{155}$ Wilmoth, R. G., Mitcheltree, R. A., and Moss, J. N., "Low-Density Aerodynamics of the Stardust Sample Return Capsule," AIAA 97-2510, AIAA, June 1997.

${ }^{156}$ Desai, P. N., Mitcheltree, R. A., and Cheatwood, F. M., "Entry Dispersion Analysis for the Stardust Sample Return Capsule," AIAA 97-3812, AIAA, Aug. 1997.

${ }^{157}$ Chen, Y. K. and Milos, F. S., "Fully Implicit Ablation and Thermal Analysis Program (FIAT)," Fourth International Conference on Composites and Engineering, July 1997.

${ }^{158}$ Givens, J. J., Nolte, L. J., and Pochettino, L. R., "Galileo Atmospheric Entry Probe System: Design, Development and Test," AIAA 83-0098, AIAA, January 1983.

${ }^{159}$ Seiff, A., Kirk, B. D., Knight, T. C. D., Young, L. A., Milos, F. S., Venkatapahty, E., Mihalov, J. D., Blanchard, R. C., Young, R. E., and Shubert, G., "Thermal Structure of Jupiter's Upper Atmosphere Derived from the Galileo Probe," Science, Vol. 276, No. 4, April 1977, pp. 102-104.

${ }^{160}$ Moss, J. N. and Simmonds, A. L., "Galileo Probe Forebody Flowfield Predictions," Entry Vehicle Heating and Thermal Protection Systems: Space Shuttle, Solar Starprobe, Galileo Probe, edited by P. E. Bauer and H. E. Collicott, Vol. 85 of Progress in Astronautics and Aeronautics, AIAA, 1983, pp. 419-445, (See also AIAA Paper 82-0874).

${ }^{161}$ Green, M. J. and Davy, W. C., "Galileo Probe Forebody Thermal Protection," Thermophysics of Atmospheric Entry, edited by T. E. Horton, Vol. 82 of Progress in Astronautics and Aeronautics, AIAA, 1982, pp. 328-353, (See also AIAA Paper 81-1073).

${ }^{162}$ Howe, J. T., Pitts, W. C., and Lundell, J. H., "Survey of the Supporting Research and Technology for the Thermal Protection of the Galileo Probe," Thermophysics of Atmospheric Entry, edited by T. E. Horton, Vol. 82 of Progress in Astronautics and Aeronautics, AIAA, 1982, pp. 293-327, (See also AIAA Paper 81-1068).

${ }^{163}$ Seiff, A., Venkatapahty, E., Hass, B., and Intrieri, P., "Galileo Probe Aerodynamics," AIAA 96-2451, AIAA, June 1996.

${ }^{164}$ Maus, J. R., Griffith, B. J., Szema, K. Y., and Best, J. T., "Hypersonic Mach Number and Real Gas Effects of Space Shuttle Orbiter Aerodynamics," AIAA 83-0343, AIAA, January 1983

${ }^{165}$ Slotnick, J. P., Kandula, M., Buning, P. G., and F. W. Martin, J., "Numerical Simulation of the Space Shuttle Launch Vehicle with Real Gas Solid Rocket Plume Effects," AIAA 93-0521, AIAA, January 1993.

${ }^{166}$ Engle, C. D. and Praharaj, S. C., "MINIVER Upgrade for the AVID System, Vol. I: LANMIN Users Manual," CR 172212, NASA, Hampton VA 23681-0001, August 1983.

${ }^{167}$ Bowles, J. V., "Conceptual Design Studies at NASA Ames," Second NASP Technology Syposium, October 1986, NASP-CP-2086, APL, John Hopkins University, Laurel Maryland.

${ }^{168}$ Höld, R. K. and Fornasier, L., "Investigation of Thermal Loads on Hypersonic Vehicles with Emphasis on Surface Radiation Effects," ICAS Proceedings 1994, Vol. 2, September 1994, pp. $1172-1182$.

${ }^{169}$ Horvath, T. J., McGinley, C. B., and Hannemann, K., "Blunt Body Near Wake Flow Field at Mach 6," AIAA 96-1935, AIAA, June 1996.

${ }^{170}$ Simeonides, G., Walpot, L., Netterfield, M., and Tumino, G., "Evaluation of Engineering Heat Transfer Prediction Methods in High Enthalpy Flow Conditions," AIAA 96-1860, AIAA, June 1996.

${ }^{171}$ Gnoffo, P. A. and Inger, G. R., "Analytic Corrections to CFD Heating Predictions Accounting for Changes in Surface Catalysis," AIAA 96-1800, AIAA, June 1996.
172 Gnoffo, P. A. and Inger, G. R., "Analytic Corrections to CFD Heating Predictions Accounting for Changes in Surface Catalysis, Part II," AIAA 96-4589, AIAA, November 1996.

${ }^{173}$ Hamilton II, H. H., Greene, F. A., and DeJarnette, F. R., "Approximate Method for Calculating Heating Rates on Three-Dimensional Vehicles," Journal of Spacecraft and Rockets, Vol. 31, No. 1, May-June 1994, pp. 345-354.

${ }^{174}$ Zoby, E. V., Moss, J. N., and Sutton, K., "Approximate Convective Heating Analysis for Hypersonic Flows," Journal of Spacecraft and Rockets, Vol. 18, No. 1, January-February 1981, pp. 64-70.

${ }^{175}$ Murray, A. L., "Further Enhancements of the BLIMP Computer Code and Users Guide," TR 3010, AFWAL, June 1988.

${ }^{176}$ Ganguly, B. N. and Bletzinger, P., "Shock Wave Dispersion in Nonequilibrium Plasmas," AIAA 96-4607, AIAA, November 1996. 